\title{
A new approach to strongly correlated fermion systems: the spin-particle-hole coherent-state path integral
}

\author{
N. Dupuis \\ Laboratoire de Physique des Solides, Associé au CNRS, Université Paris-Sud, 91405 Orsay, France
}

(May 3, 2001)

\begin{abstract}
We describe a new path integral approach to strongly correlated fermion systems, considering the Hubbard model as a specific example. Our approach is based on the introduction of spinparticle-hole coherent states which generalize the spin- $\frac{1}{2}$ coherent states by allowing the creation of a hole or an additional particle. The action of the fermion system $S\left[\gamma^{*}, \gamma ; \boldsymbol{\Omega}\right]$ can be expressed as a function of two Grassmann variables $\left(\gamma_{\uparrow}, \gamma_{\downarrow}\right)$ describing particles propagating in the lower and upper Hubbard bands, and a unit vector field $\boldsymbol{\Omega}$ whose dynamics arises from spin fluctuations. In the strong correlation limit, $S\left[\gamma^{*}, \gamma ; \Omega\right]$ can be truncated to quartic order in the fermionic fields and used as the starting point of a strong-coupling diagrammatic expansion in $t / U$ ( $t$ being the intersite hopping amplitude and $U$ the on-site Coulomb repulsion). We discuss possible applications of this formalism and its connection to the $t$ - $J$ model and the spin-fermion model.
\end{abstract}

PACS Numbers: 71.10.Fd, 71.27.+a, 71.30+h

\section{INTRODUCTION}

Understanding the properties of strongly correlated fermion systems remains one of the main goals of condensed-matter physics. In narrow-band electron systems, the interplay between strong Coulomb repulsion (which tends to localize the electrons) and band structure effects (which favor their itinerant character) leads to a variety of different behaviors ranging from metallic Fermi liquid to Mott-Hubbard insulator. Standard weakcoupling approaches fail to describe these phenomena so that no general theoretical method is available to analyze strongly correlated fermion systems. Even for the Hubbard model, 10 which is supposed to be one of the simplest (realistic) models of strongly correlated fermions, exact solutions or well-controlled approximations exist only in a few special cases, like in one-dimensions or in the limit of infinite dimensions. 6 We describe in this paper a new approach to strongly correlated systems, with the (two-dimensional) Hubbard model as primary example.

Various weak-coupling theories have been applied to the Hubbard model and its extensions. The simplest of these approaches, the Hartree-Fock theory, supplemented by the random-phase approximation (RPA) for the calculation of susceptibilities, correctly predicts an antiferromagnetic (AF) ground-state at half-filling in both limits of large and small on-site Coulomb repulsion $U$ However, it also predicts long-range $\mathrm{AF}$ order at finite temperature in a two-dimensional (2D) system, in contradiction with Mermin-Wagner theorem. Many attempts to improve on Hartree-Fock/RPA theory can be ferund in the literature: paramagnonlike thepries 9 - 10 fluctuation exchange approximation (FLEX) 11, 12 pseudo-potential Parquet approach, 21,13 two-particle self-consistent theory. ${ }^{4}$ Most of these approaches meet with serious difficulties regarding a correct description of the physical properties of the 2D Hubbard model: absence of long-range order at finite temperature, exponential divergence of the magnetic correlation length in $1 / T$ at low temperature, existence of Hubbard bands and/or precursors of the AF bands in the density of states, pseudo-gap at low energy, etc. Besides, irrespective of their success at weak Coulomb repulsion, it seems that weak-coupling theories are doomed to fail in the strong-coupling regime when the Coulomb repulsion exceeds the bandwidth $(U \sim 4 D t$ with $t$ the intersite hopping amplitude and $D$ the dimensionality). [See Ref. 14 for a detailed discussion of weak-coupling approaches to the Hubbard model.] As emphasized early on by Mott, 15 a characteristic property of strongly correlated systems is the existence of local moments already in the metallic phase 4 Weak-coupling theories fail to properly describe these local moments. For instance, within Hartree-Fock theory, local moments are totally absent in the metallic phase and appear only in presence of long-range magnetic order.

On the other hand the perturbation expansion around the atomic limit, 1624 which is expected to provide a reliable starting point in the strong correlation limit, also presents its own difficulties in spite of recent progress.2526 It is not completely clear how to handle the degeneracy of the ground-state in the atomic limit.2 Moreover, the expansion involves two dimensionless parameters, $t / U$ and $t / T$, and therefore breaks down when the temperature is much below the electronic bandwidth.

Nonetheless, it is possible to derive an effective Hamiltonian to a given order in $t / U 2729$ At half-filling, the Hubbard model reduces to the Heisenberg model in the strong-coupling limit. This model describes local spins (i.e. local moments) coupled by short-range exchange interactions. Away from half-filling, the effective Hamiltonian in the strong correlation limit is given by the $t$ $J$ model. So far, no satisfying description of a doped Mott insulator has emerged from the $t-J$ model. The 
Schwinger-boson slave-fermion mean-field theory, which provides very good results at halffilling, 30 has not been as successful in the doped case. $\frac{31.32}{32}$ On the other hand, the slave-boson mean-field theory 33 does not even reproduce the known results at half-filling, so that its predictions (near half-filling) are highly questionable.

A very appealing path integral formulation of the $t-J$ model, based on the spin-hole coherent-states $|\boldsymbol{\Omega}, \zeta\rangle$, has been introduced by Auerbach and Larson. 34 The existence of local moments is obvious in this formulation, since a singly occupied site $\mathbf{r}$ is described by a spincoherent state $\left|\boldsymbol{\Omega}_{\mathbf{r}}\right\rangle$. The set of Grassmann variables $\zeta=\left\{\zeta_{\mathbf{r}}\right\}$ allows for the presence of holes. The spinhole coherent-state path integral was used to study spin polarons in the (large- $S$ ) semiclassical limit of the $t-J$ model.

Schulz has derived a path integral formulation of the Hubbard modelyhich also exemplifies the existence of local moments 35 . 36 The Coulomb repulsion is treated within a large- $U$ Hartree-Fock approximation, whereas the $\mathrm{SU}(2)$ spin-rotation symmetry is maintained by introducing a fluctuating spin-quantization axis in the functional integral. The resulting effective action $S\left[\gamma^{*}, \gamma ; \boldsymbol{\Omega}\right]$ is expressed in terms of two Grassmann variables $\left(\gamma_{\uparrow}, \gamma_{\downarrow}\right)$ which describe particles propagating in the lower (LHB) anfupper (UHB) Hubbard bands, and a unit vector field $\boldsymbol{\Omega} .37$ A singly occupied site $\mathbf{r}$ corresponds to a local moment pointing in the direction of $\boldsymbol{\Omega}_{\mathbf{r}}$. Similar ideas, in view of Monte Carlo simulation, have been recently discussed by Bickers and Scalapino 38

In a previous publication, Pairault and the present author have reported a systematic $t / U$ expansion of the Hubbard model. 39 As in Schulz's work, central to this approach is the introduction of a fluctuating spin-quantization axis. By adapting the method of Refs. 40,25, the $t / U$ expansion was generated by a Grassmannian Hubbard-Stratonovich transformation of the hopping term. As in Ref. 35, the effective action $S\left[\gamma^{*}, \gamma ; \boldsymbol{\Omega}\right]$ of the Hubbard model in the strong-coupling regime is expressed as a function of two Grassmann variables and a unit vector field. We found however that the Hartree-Fock treatment of the Coulomb repulsion is not sufficient in the strong correlation limit, since it misses processes of order $t / U$. In particular, it does not allow to recover the $t-J$ model when the UHB is integrated out. 39

The aim of this paper is to further develop the strongcoupling expansion introduced in Ref. 39. (i) The functional integral formulation is derived from a completely different perspective, starting from spin-particle-hole coherent states (Sec. II). The latter generalize the spin- $\frac{1}{2}$ coherent states by allowing the creation of a hole or the introduction of an additional particle. This new derivation emphasizes the connection with the spin coherentstate path integral formulation of the Heisenberg model. (ii) As in Ref. 39, the strong-coupling expansion is generated by two successive Hubbard-Stratonovich transformations of the intersite hopping term. The resulting action $S\left[\gamma^{*}, \gamma ; \boldsymbol{\Omega}\right]$ is obtained to all orders in $t / U$
(Sec. III A). This action contains interaction terms to all orders which are determined by the (exact) atomic vertices. In the strong correlation limit, $S\left[\gamma^{*}, \gamma ; \boldsymbol{\Omega}\right]$ can be truncated to quartic order in the fermionic fields (Sec. III Q). The action is then entirely determined by the single-particle atomic Green's function and the two-particle atomic vertex. (iii) We discuss in detail the strong-coupling perturbative expansion and briefly point out possible applications of our formalism which are developed in detail in separate publications 41,42 (Sec. III D). Finally, we discuss the connection of this formalism to the $t-J$ model and the spin-fermion model.

\section{SPIN-PARTICLE-HOLE COHERENT-STATE PATH INTEGRAL}

In this section, we define the spin-particle-hole coherent states and derive a path integral representation of the partition function. To be specific, we consider the Hubbard model defined by

$$
\hat{H}=-t \sum_{\left\langle\mathbf{r}, \mathbf{r}^{\prime}\right\rangle, \sigma}\left(\hat{c}_{\mathbf{r} \sigma}^{\dagger} \hat{c}_{\mathbf{r}^{\prime} \sigma}+\text { h.c. }\right)+U \sum_{\mathbf{r}} \hat{n}_{\mathbf{r} \uparrow} \hat{n}_{\mathbf{r} \downarrow}
$$

where $\hat{c}_{\mathbf{r} \sigma}$ is a fermionic operator for a $\sigma$-spin particle at site $\mathbf{r}(\sigma=\uparrow, \downarrow), \hat{n}_{\mathbf{r} \sigma}=\hat{c}_{\mathbf{r} \sigma}^{\dagger} \hat{c}_{\mathbf{r} \sigma}$, and $\left\langle\mathbf{r}, \mathbf{r}^{\prime}\right\rangle$ denotes nearest neighbors. For simplicity, we consider a bipartite $D$-dimensional lattice. We denote by $\mu$ the chemical potential and consider only hole doping (i.e. $\mu \leq U / 2$ ). We take $\hbar=k_{B}=1$ throughout the paper.

\section{A. Spin-particle-hole coherent-states}

\section{Definition}

We first consider a single site. A basis of the Hilbert space is $\mathcal{B}=\{|0\rangle,|\uparrow\rangle,|\downarrow\rangle,|\uparrow \downarrow\rangle\}$, where $|0\rangle,|\uparrow\rangle=\hat{c}_{\uparrow}^{\dagger}|0\rangle$, $|\downarrow\rangle=\hat{c}_{\downarrow}^{\dagger}|0\rangle$ and $|\uparrow \downarrow\rangle=\hat{c}_{\uparrow}^{\dagger} \hat{c}_{\downarrow}^{\dagger}|0\rangle$ are the empty, singly occupied (with spin up and spin down) and doubly occupied states, respectively. Instead of using the states $|\uparrow\rangle$ and $|\downarrow\rangle$ to describe a singly occupied site, we introduce the spin- $\frac{1}{2}$ coherent state

$$
\begin{aligned}
|\boldsymbol{\Omega}\rangle & =e^{-\frac{i}{2} \varphi \sigma_{z}} e^{-\frac{i}{2} \theta \sigma_{y}} e^{-\frac{i}{2} \psi \sigma_{z}}|\uparrow\rangle \\
& =\cos \frac{\theta}{2} e^{-\frac{i}{2}(\varphi+\psi)}|\uparrow\rangle+\sin \frac{\theta}{2} e^{\frac{i}{2}(\varphi-\psi)}|\downarrow\rangle,
\end{aligned}
$$

where $\theta$ and $\varphi$ are the polar angles determining the direction of the unit vector $\boldsymbol{\Omega}(\theta, \varphi)$. The shoice of $\psi$ is free and corresponds to a "gauge" freedom.43 $\mathcal{B}_{\boldsymbol{\Omega}}^{\prime}=\{|0\rangle,|\boldsymbol{\Omega}\rangle, \mid \uparrow \downarrow$ \rangle$\}$ will be used to construct the spin-particle-hole coherent states. Although it is not (for fixed $\boldsymbol{\Omega}$ ) a basis of the Hilbert space, we will show how the whole Hilbert space can be described by varying $\boldsymbol{\Omega}$. 
We now enlarge the physical Hilbert space by introducing three bosonic operators $(\hat{e}, \hat{p}$ and $\hat{d})$ and rewrite the states of $\mathcal{B}_{\Omega}^{\prime}$ as

$$
\begin{aligned}
|0\rangle & =\hat{e}^{\dagger}|\operatorname{vac}\rangle=\hat{\gamma}_{\uparrow}|\boldsymbol{\Omega}\rangle, \\
|\boldsymbol{\Omega}\rangle & =\hat{p}^{\dagger} \hat{f}_{\uparrow}^{\dagger}|\mathrm{vac}\rangle, \\
|\uparrow \downarrow\rangle & =\hat{d}^{\dagger} \hat{f}_{\uparrow}^{\dagger} \hat{f}_{\downarrow}^{\dagger}|\mathrm{vac}\rangle=-\hat{\gamma}_{\downarrow}^{\dagger}|\boldsymbol{\Omega}\rangle,
\end{aligned}
$$

where $\mid$ vac $\rangle$ denotes the vacuum of the enlarged Hilbert space. The latter contains unphysical states which can be eliminated by imposing the constraints

$$
\begin{aligned}
& \hat{Q}^{(1)}=\hat{e}^{\dagger} \hat{e}+\hat{p}^{\dagger} \hat{p}+\hat{d}^{\dagger} \hat{d}-1=0, \\
& \hat{Q}_{\uparrow}^{(2)}=\hat{f}_{\uparrow}^{\dagger} \hat{f}_{\uparrow}-\hat{p}^{\dagger} \hat{p}-\hat{d}^{\dagger} \hat{d}=0, \\
& \hat{Q}_{\downarrow}^{(2)}=\hat{f}_{\downarrow}^{\dagger} \hat{f}_{\downarrow}-\hat{d}^{\dagger} \hat{d}=0 .
\end{aligned}
$$

The (slave) bosonic particles are similar to those introduced by Kotliar and Ruckenstein 44 although here the $\hat{p}$ boson is spinless. Once we have introduced the spin$\frac{1}{2}$ coherent state $|\boldsymbol{\Omega}\rangle$, we need two operators, $\hat{\gamma}_{\uparrow}$ and $\hat{\gamma}_{\downarrow}$, to allow for the creation of a hole or an additional particle. These two operators are Hubbard operators 45 $\left(\hat{\gamma}_{\uparrow} \equiv|0\rangle\langle\boldsymbol{\Omega}|\right.$ and $\left.\hat{\gamma}_{\downarrow} \equiv-|\boldsymbol{\Omega}\rangle\langle\uparrow \downarrow|\right)$, and have the following expression,

$$
\begin{aligned}
& \hat{\gamma}_{\uparrow}=\hat{e}^{\dagger} \hat{p} \hat{f}_{\uparrow}, \\
& \hat{\gamma}_{\downarrow}=\hat{p}^{\dagger} \hat{d} \hat{f}_{\downarrow}
\end{aligned}
$$

in terms of the slave bosons and the fermionic operators $\hat{f}_{\sigma}$. Note that all the operators are defined with respect to the spin-quantization axis $\boldsymbol{\Omega}$, so that a $\hat{f}_{\uparrow^{-}}$( or $\hat{\gamma}_{\uparrow^{-}}$) particle has its spin pointing along $\boldsymbol{\Omega}$. Thus, the LHB is populated only with up-spin particles (in the spin reference frame defined by $\boldsymbol{\Omega}$ ). This also implies that only down-spin particles can be introduced in the UHB. The operators $\hat{\gamma}_{\sigma}$ play a fundamental role in our approach. Although they appear at this stage as composite operators [see Eqs. (2.5)], we shall show in Sec. IIII how the partition function can be written as a functional integral expressed in terms of the elementary fields $\gamma_{\sigma}$ and the unit vector field $\boldsymbol{\Omega}$.

The spin-particle-hole coherent states are defined by

$$
|\boldsymbol{\Omega}, \zeta\rangle=\exp \left(e \hat{e}^{\dagger}+p \hat{p}^{\dagger}+d \hat{d}^{\dagger}-f_{\uparrow} \hat{f}_{\uparrow}^{\dagger}-f_{\downarrow} \hat{f}_{\downarrow}^{\dagger}\right)|\mathrm{vac}\rangle,
$$

where $f_{\uparrow}, f_{\downarrow}$ are Grassmann variables and $e, p, d c$ numbers. We use the short-hand notation $\zeta \equiv$ $\left(f_{\uparrow}, f_{\downarrow}, e, p, d\right)$. By using the constraints (2.4), we can rewrite the spin-particle-hole coherent states as

$$
|\boldsymbol{\Omega}, \zeta\rangle=e|0\rangle-p f_{\uparrow}|\boldsymbol{\Omega}\rangle-d f_{\uparrow} f_{\downarrow}|\uparrow \downarrow\rangle .
$$

Eqs. (2.6) and (2.7) extend the definition of the spin- $\frac{1}{2}$ coherent states by allowing for the presence of a hole or an additional particle.

\section{Scalar product}

We consider the scalar product between the states $|\boldsymbol{\Omega}, \zeta\rangle$ and $\left|\boldsymbol{\Omega}^{\prime}, \zeta^{\prime}\right\rangle$. If $\boldsymbol{\Omega}=\boldsymbol{\Omega}^{\prime}$ all fermionic variables are defined with respect to the same quantization axis. We then have the standard result 46

$$
\begin{aligned}
\left\langle\boldsymbol{\Omega}, \zeta \mid \boldsymbol{\Omega}^{\prime}, \zeta^{\prime}\right\rangle & =\exp \left(\zeta^{*} \zeta^{\prime}\right) \\
& \equiv \exp \left(e^{*} e^{\prime}+p^{*} p^{\prime}+d^{*} d^{\prime}+\sum_{\sigma} f_{\sigma}^{*} f_{\sigma}^{\prime}\right) .
\end{aligned}
$$

When $\boldsymbol{\Omega} \neq \boldsymbol{\Omega}^{\prime}$, we use Eq. (2.7) to obtain

$$
\begin{aligned}
\left\langle\boldsymbol{\Omega}, \zeta \mid \boldsymbol{\Omega}^{\prime}, \zeta^{\prime}\right\rangle= & e^{*} e^{\prime}+p^{*} f_{\uparrow}^{*} f_{\uparrow}^{\prime} p^{\prime}+d^{*} d^{\prime} f_{\downarrow}^{*} f_{\uparrow}^{*} f_{\uparrow}^{\prime} f_{\downarrow}^{\prime} \\
& +p^{*} f_{\uparrow}^{*} f_{\uparrow}^{\prime} p^{\prime}\left(\left\langle\boldsymbol{\Omega} \mid \boldsymbol{\Omega}^{\prime}\right\rangle-1\right) .
\end{aligned}
$$

For $\boldsymbol{\Omega}=\boldsymbol{\Omega}^{\prime}$, this expression should be equivalent to Eq. 2.8), so that

$$
\exp \left(\zeta^{*} \zeta^{\prime}\right) \equiv e^{*} e^{\prime}+p^{*} f_{\uparrow}^{*} f_{\uparrow}^{\prime} p^{\prime}+d^{*} d^{\prime} f_{\downarrow}^{*} f_{\uparrow}^{*} f_{\uparrow}^{\prime} f_{\downarrow}^{\prime}
$$

provided the constraints (2.4) are satisfied. Similarly, by keeping only the terms which are compatible with the constraints, we obtain

$$
\begin{array}{r}
\exp \left(\zeta^{*} \zeta^{\prime}+f_{\uparrow}^{*} p^{*} f_{\uparrow}^{\prime} p^{\prime}\left(\left\langle\boldsymbol{\Omega} \mid \boldsymbol{\Omega}^{\prime}\right\rangle-1\right)\right)=e^{*} e^{\prime} \\
+p^{*} f_{\uparrow}^{*} f_{\uparrow}^{\prime} p^{\prime}\left\langle\boldsymbol{\Omega} \mid \boldsymbol{\Omega}^{\prime}\right\rangle+d^{*} d^{\prime} f_{\downarrow}^{*} f_{\uparrow}^{*} f_{\uparrow}^{\prime} f_{\downarrow}^{\prime},
\end{array}
$$

a result valid whatever the value of $\boldsymbol{\Omega}$ and $\boldsymbol{\Omega}^{\prime}$. Comparing Eqs. (2.9) and (2.11), we arrive at the following expression for the scalar product of two spin-particle-hole coherent states in the physical Hilbert space:

$$
\left\langle\boldsymbol{\Omega}, \zeta \mid \boldsymbol{\Omega}^{\prime}, \zeta^{\prime}\right\rangle=\exp \left(\zeta^{*} \zeta^{\prime}+f_{\uparrow}^{*} p^{*} f_{\uparrow}^{\prime} p^{\prime}\left(\left\langle\boldsymbol{\Omega} \mid \boldsymbol{\Omega}^{\prime}\right\rangle-1\right)\right) .
$$

\section{Resolution of the identity}

We seek a resolution of the identity in the form

$$
\mathcal{N} \int \frac{d \boldsymbol{\Omega}}{4 \pi} \int d \zeta^{*} d \zeta e^{-\alpha|\zeta|^{2}}|\boldsymbol{\Omega}, \zeta\rangle\langle\boldsymbol{\Omega}, \zeta| \hat{P}=\hat{I},
$$

where $\hat{I}$ is the unit operator and

$$
\alpha|\zeta|^{2} \equiv \alpha_{e}|e|^{2}+|p|^{2}+\alpha_{d}|d|^{2}+\sum_{\sigma} f_{\sigma}^{*} f_{\sigma} .
$$

$\mathcal{N}, \alpha_{e}$ and $\alpha_{d}$ are constants to be determined in order for Eq. (2.13) to be satisfied. The projection operator

$$
\hat{P}=\delta_{\hat{Q}^{(1)}, 0} \prod_{\sigma} \delta_{\hat{Q}_{\sigma}^{(2)}, 0}
$$

ensures that the resolution of the identity acts only in the physical Hilbert space. The measure in Eq. (2.13) is defined by 


$$
d \zeta^{*} d \zeta=\frac{d e^{*} d e}{2 i \pi} \frac{d p^{*} d p}{2 i \pi} \frac{d d^{*} d d}{2 i \pi} d f_{\uparrow}^{*} d f_{\uparrow} d f_{\downarrow}^{*} d f_{\downarrow}
$$

where

$$
\frac{d z^{*} d z}{2 i \pi}=\frac{1}{\pi} d \operatorname{Re}(z) d \operatorname{Im}(z)
$$

for a complex variable $z$. Integrating over $\zeta$, we rewrite Eq. (2.13) as

$$
\mathcal{N} \int \frac{d \boldsymbol{\Omega}}{4 \pi}\left(\frac{|0\rangle\langle 0|}{\alpha_{e}^{2} \alpha_{d}}+\frac{|\boldsymbol{\Omega}\rangle\langle\boldsymbol{\Omega}|}{\alpha_{e} \alpha_{d}}+\frac{|\uparrow \downarrow\rangle\langle\uparrow \downarrow|}{\alpha_{e} \alpha_{d}^{2}}\right)=\hat{I} .
$$

Since the spin coherent states satisfy $2(4 \pi)^{-1} \int d \boldsymbol{\Omega}|\boldsymbol{\Omega}\rangle\langle\boldsymbol{\Omega}|=$ $|\uparrow\rangle\langle\uparrow|+| \downarrow\rangle\langle\downarrow|$ (resolution of the identity in the space of singly occupied states), we conclude that Eq. (2.13) is satisfied for

$$
\begin{aligned}
& \mathcal{N}=8, \\
& \alpha_{e}=\alpha_{d}=2 .
\end{aligned}
$$

The constants $\alpha_{e}=\alpha_{d}(\neq 1)$ are necessary to avoid an over-counting of the empty and doubly occupied states: for a given $\boldsymbol{\Omega}, \mathcal{B}_{\boldsymbol{\Omega}}^{\prime}$ already contains the states $|0\rangle$ and $|\uparrow \downarrow\rangle$. Varying $\boldsymbol{\Omega}$ with $\alpha_{e}=\alpha_{d}=1$ would lead to an over-counting of these states.

Note that Eq. (2.13) bears some similarities with the resolution of the identity in the Hilbert space with no double-gefupancy obtained from the spin-hole coherent states. 13.34

\section{Trace}

The resolution of the identity (2.13) allows to compute the trace of a given operator $\hat{O}$ in terms of the spin-particle-hole coherent states. The derivation is standard 46 and one obtains

$$
\operatorname{Tr} \hat{O}=\mathcal{N} \int \frac{d \boldsymbol{\Omega}}{4 \pi} \int d \zeta^{*} d \zeta e^{-\alpha|\zeta|^{2}}\langle\boldsymbol{\Omega}, \tilde{\zeta}|\hat{P} \hat{O}| \boldsymbol{\Omega}, \zeta\rangle
$$

where $\tilde{\zeta} \equiv\left(-f_{\uparrow},-f_{\downarrow}, e, p, d\right)$.

As a simple application of Eq. (2.20), we calculate the partition function for a single site. Imposing the constraints (2.4) by using Eq. (2.7), we easily obtain

$$
\begin{aligned}
Z_{\text {at }} & =\operatorname{Tr} e^{-\beta(\hat{H}-\mu \hat{N})} \\
& =\mathcal{N}\left(\frac{1}{\alpha_{e}^{2} \alpha_{d}}+\frac{e^{\beta \mu}}{\alpha_{e} \alpha_{d}}+\frac{e^{\beta(2 \mu-U)}}{\alpha_{e} \alpha_{d}^{2}}\right) \\
& =1+2 e^{\beta \mu}+e^{\beta(2 \mu-U)},
\end{aligned}
$$

where $\hat{N}$ is the total number of particles and $\beta=1 / T$ is the inverse temperature.

\section{B. Path integral for a single site}

In order to express the partition function as a path integral, we use Eq. (2.20) and divide the "time" interval $\beta$ into $M$ steps:

$$
\begin{aligned}
Z_{\text {at }}= & \mathcal{N} \int \frac{d \boldsymbol{\Omega}}{4 \pi} \int d \zeta^{*} d \zeta e^{-\alpha|\zeta|^{2}} \\
& \times\left\langle\boldsymbol{\Omega}, \tilde{\zeta}\left|\hat{P} e^{-\epsilon(\hat{H}-\mu \hat{N})} \cdots e^{-\epsilon(\hat{H}-\mu \hat{N})}\right| \boldsymbol{\Omega}, \zeta\right\rangle,
\end{aligned}
$$

where $\epsilon=\beta / M$. We then introduce $(M-1)$ times the resolution of the identity (2.13). The details of this procedure are given in Appendix A. One finds

$$
\begin{aligned}
Z_{\mathrm{at}}= & \mathcal{N}^{M} \int\left(\prod_{k=1}^{M} \frac{d \boldsymbol{\Omega}_{k}}{4 \pi} d \zeta_{k}^{*} d \zeta_{k} d \lambda_{k}\right) \\
& \times \exp \left\{\sum _ { k = 1 } ^ { M } \left[-\alpha\left|\zeta_{k}\right|^{2}+\zeta_{k}^{*} \zeta_{k-1}\right.\right. \\
& \left.\left.+f_{\uparrow k}^{*} p_{k}^{*} f_{\uparrow k-1} p_{k-1}\left(\left\langle\boldsymbol{\Omega}_{k} \mid \boldsymbol{\Omega}_{k-1}\right\rangle-1\right)-\epsilon K_{k, k-1}\right]\right\},
\end{aligned}
$$

where

$$
\begin{aligned}
K_{k, k-1} & =\frac{\left\langle\boldsymbol{\Omega}_{k}, \zeta_{k}\left|\hat{K}_{k}\right| \boldsymbol{\Omega}_{k-1}, \zeta_{k-1}\right\rangle}{\left\langle\boldsymbol{\Omega}_{k}, \zeta_{k} \mid \boldsymbol{\Omega}_{k-1}, \zeta_{k-1}\right\rangle}, \\
\hat{K}_{k} & =\hat{H}-\mu \hat{N}+i \lambda_{k}^{(1)} \hat{Q}_{k}^{(1)}+i \sum_{\sigma} \lambda_{\sigma k}^{(2)} \hat{Q}_{\sigma}^{(2)} .
\end{aligned}
$$

$\lambda_{k} \equiv\left(\lambda_{k}^{(1)}, \lambda_{\sigma k}^{(2)}\right)$ denotes Lagrange multipliers which impose the constraints (2.4).

An important difference with the standard path integral for a system of fermions and bosons comes from the presence of the constants $\alpha_{e}=\alpha_{d}(\neq 1)$ which ensure that the empty and doubly occupied states are not overcounted. Taking the continuum time limit $(\epsilon \rightarrow 0)$ from Eq. (2.23) would then lead to an infinite chemical potential $\mu_{e}=-\left(\alpha_{e}-1\right) / \epsilon$ for the $e$ boson:

$$
\sum_{k=1}^{M} e_{k}^{*}\left(e_{k-1}-\alpha_{e} e_{k}\right) \rightarrow-\int_{0}^{\beta} d \tau e^{*}\left(\partial_{\tau}+\frac{\alpha_{e}-1}{\epsilon}\right) e
$$

and a similar result for the $d$ boson (i.e. $\mu_{d}=-\left(\alpha_{d}-\right.$ $1) / \epsilon)$.

At half-filling, $\mu=U / 2$ due to particle-hole symmetry. The contribution of the empty and doubly occupied states to the partition function is exponentially small at low temperature $(T \ll U / 2)$ and can be neglected. It is then possible to take $\alpha_{e}=\alpha_{d}=1$, and the continuum time limit is well defined. This argument fails away from half-filling in the single-site case. However, we are interested in the full Hubbard model, where the chemical potential is determined by the filling of the Hubbard bands in the strong correlation limit. In presence of a 
small concentration of holes, $\mu \simeq W / 2$ is located near the top of the LHB (of total width $W$ ). In the lowtemperature limit $T \ll W$, the system in the atomic limit is in its ground-state with exactly one particle per site. The contribution of the empty and doubly occupied atomic states to the partition function is again negligible, which allows to take $\alpha_{e}=\alpha_{d}=1$. This argument is particularly clear in the strong-coupling expansion (Sec. III) where the atomic action is used only in the calculation of the connected atomic functions $G^{R c}$. The latter are obtained from the atomic limit $(t=0)$ of the full action, the chemical potential being determined by the full Hubbard model.

Taking $\alpha_{e}=\alpha_{d}=1$ and ignoring the overall normalization constant $\mathcal{N}^{M} 4$ we write the partition function as

$$
\begin{aligned}
Z_{\mathrm{at}}= & \int\left(\prod_{k=1}^{M} \frac{d \boldsymbol{\Omega}_{k}}{4 \pi} d \zeta_{k}^{*} d \zeta_{k} d \lambda_{k}\right) \exp \left\{-\sum_{k=1}^{M}\left[\zeta_{k}^{*}\left(\zeta_{k}-\zeta_{k-1}\right)\right.\right. \\
& \left.\left.-f_{\uparrow k}^{*} p_{k}^{*} f_{\uparrow k-1} p_{k-1}\left(\left\langle\boldsymbol{\Omega}_{k} \mid \boldsymbol{\Omega}_{k-1}\right\rangle-1\right)+\epsilon K_{k, k-1}\right]\right\} .
\end{aligned}
$$

The continuum time limit can now be taken without difficulty (see Appendix A):

$$
Z_{\text {at }}=\int \mathcal{D} \boldsymbol{\Omega} \int \mathcal{D} \lambda \int \mathcal{D}[f, e, p, d] e^{-S_{\mathrm{at}}},
$$

where the action is given by

$$
\begin{aligned}
S_{\mathrm{at}}= & S_{\mathrm{at}}^{(0)}+\int d \tau f_{\uparrow}^{*} p^{*} f_{\uparrow} p\langle\boldsymbol{\Omega} \mid \dot{\boldsymbol{\Omega}}\rangle \\
S_{\mathrm{at}}^{(0)}= & \int d \tau\left[-i \lambda^{(1)}+\sum_{\sigma} f_{\sigma}^{*}\left(\partial_{\tau}-\mu+i \lambda_{\sigma}^{(2)}\right) f_{\sigma}\right. \\
& +e^{*}\left(\partial_{\tau}+i \lambda^{(1)}\right) e+p^{*}\left(\partial_{\tau}+i \lambda^{(1)}-i \lambda_{\uparrow}^{(2)}\right) p \\
& \left.+d^{*}\left(\partial_{\tau}+U+i \lambda^{(1)}-i \sum_{\sigma} \lambda_{\sigma}^{(2)}\right) d\right] .
\end{aligned}
$$

$S_{\text {at }}^{(0)}$ is similar to the actign obtained from the KotliarRuckenstein slave bosons 44 with $p_{\uparrow} \equiv p$ and without the $p_{\downarrow}$ boson. The difference between $S_{\text {at }}$ and $S_{\text {at }}^{(0)}$, which arises from the dynamics of $\boldsymbol{\Omega}$, is nothing but the Berry phase term $A^{0}=\langle\boldsymbol{\Omega} \mid \dot{\boldsymbol{\Omega}}\rangle$ of a spin- $\frac{1}{2}\left(|\dot{\boldsymbol{\Omega}}\rangle=\partial_{\tau}|\boldsymbol{\Omega}\rangle\right)$. The Berry phase term is alive whenever the state is singly occupied $\left(f_{\uparrow}^{*} f_{\uparrow} \equiv 1\right.$ and $\left.p^{*} p \equiv 1\right)$.

\section{Path integral for the Hubbard model}

The definition of the spin-particle-hole coherent states can be extended to the lattice case:

$$
|\boldsymbol{\Omega}, \zeta\rangle=\exp \left(\sum_{\mathbf{r}}\left(e_{\mathbf{r}} \hat{e}_{\mathbf{r}}^{\dagger}+p_{\mathbf{r}} \hat{p}_{\mathbf{r}}^{\dagger}+d_{\mathbf{r}} \hat{d}_{\mathbf{r}}^{\dagger}-\sum_{\sigma} f_{\mathbf{r} \sigma} \hat{f}_{\mathbf{r} \sigma}^{\dagger}\right)\right)|\operatorname{vac}\rangle
$$

where the unit vector $\boldsymbol{\Omega}_{\mathbf{r}}$ is now site dependent $[\boldsymbol{\Omega}=$ $\left\{\boldsymbol{\Omega}_{\mathbf{r}}\right\}$ and $\left.\zeta=\left\{\zeta_{\mathbf{r}}\right\}\right]$. The fermionic operator $\hat{f}_{\mathbf{r} \sigma}$ is defined with respect to the local spin-quantization axis $\boldsymbol{\Omega}_{\mathbf{r}}$.

The procedure to write the partition function as a path integral is similar to the single-site case. The only contribution which is not purely atomic comes from the intersite hopping term. The latter modifies $K_{k, k-1}$ (or, in the continuum time limit, $K_{k, k}$ ) [Eq. (2.24)]. As shown in Appendix B

$$
\left\langle\boldsymbol{\Omega}_{k}, \zeta_{k}\left|\hat{c}_{\mathbf{r}}^{\dagger} \hat{c}_{\mathbf{r}^{\prime}}\right| \boldsymbol{\Omega}_{k}, \zeta_{k}\right\rangle=\gamma_{\mathbf{r} k}^{\dagger} R_{\mathbf{r} k}^{\dagger} R_{\mathbf{r}^{\prime} k} \gamma_{\mathbf{r}^{\prime} k}\left\langle\boldsymbol{\Omega}_{k}, \zeta_{k} \mid \boldsymbol{\Omega}_{k}, \zeta_{k}\right\rangle
$$

where $\hat{c}_{\mathbf{r}}=\left(\hat{c}_{\mathbf{r} \uparrow}, \hat{c}_{\mathbf{r} \downarrow}\right)^{T}$.

$$
R_{\mathbf{r} k}=e^{-\frac{i}{2} \varphi_{\mathbf{r} k} \sigma_{z}} e^{-\frac{i}{2} \theta_{\mathbf{r} k} \sigma_{y}} e^{-\frac{i}{2} \psi_{\mathbf{r} k} \sigma_{z}}
$$

is a $\mathrm{SU}(2) / \mathrm{U}(1)$ matrix which rotates the spinquantization axis from $\hat{\mathbf{z}}$ to $\boldsymbol{\Omega}\left(\theta_{\mathbf{r} k}, \varphi_{\mathbf{r} k}\right)$. It satisfies $R_{\mathbf{r} k} \sigma_{z} R_{\mathbf{r} k}^{\dagger}=\boldsymbol{\Omega}_{\mathbf{r} k} \cdot \boldsymbol{\sigma}$, where $\boldsymbol{\sigma}=\left(\sigma_{x}, \sigma_{y}, \sigma_{z}\right)$ stands for the Pauli matrices. $\gamma_{\mathbf{r} k}=\left(\gamma_{\mathbf{r} \uparrow k}, \gamma_{\mathbf{r} \downarrow k}\right)^{T}$, and

$$
\begin{aligned}
\gamma_{\mathbf{r} \uparrow k} & =e_{\mathbf{r} k}^{*} p_{\mathbf{r} k} f_{\mathbf{r} \uparrow k}, \\
\gamma_{\mathbf{r} \downarrow k} & =p_{\mathbf{r} k}^{*} d_{\mathbf{r} k} f_{\mathbf{r} \downarrow k}
\end{aligned}
$$

The action of the Hubbard model then reads

$$
S=S_{\mathrm{at}}-\sum_{\mathbf{r}, \mathbf{r}^{\prime}} \int d \tau \gamma_{\mathbf{r}}^{\dagger} R_{\mathbf{r}}^{\dagger} t_{\mathbf{r r}^{\prime}} R_{\mathbf{r}^{\prime}} \gamma_{\mathbf{r}^{\prime}}
$$

where $S_{\text {at }}$ is the generalization to $N$ sites of the atomic action given by Eqs. (2.29). In the Hubbard model, $t_{\mathbf{r r}}$ equals $t$ if $\mathbf{r}$ and $\mathbf{r}^{\prime}$ are nearest neighbors and vanishes otherwise. Since the constraints 2.4) are preserved under the time evolution determined by the action $S$ [Eq. (2.34)], we can replace the functional integral over $\lambda(\tau)$ by an integral over a set $\lambda \equiv\left(\lambda_{\mathbf{r}}^{(1)}, \lambda_{\mathbf{r} \sigma}^{(2)}\right)$ of timeindependent Lagrange multipliers:

$$
\int \mathcal{D} \lambda \rightarrow \int d \lambda \equiv \prod_{\mathbf{r}}\left(\int_{0}^{\frac{2 \pi}{\beta}} \frac{\beta d \lambda_{\mathbf{r}}^{(1)}}{2 \pi} \prod_{\sigma} \int_{0}^{\frac{2 \pi}{\beta}} \frac{\beta d \lambda_{\mathbf{r} \sigma}^{(2)}}{2 \pi}\right) .
$$

Equations (2.29) and (2.34) provide a spin-rotationinvariant slave-boson formulation of the Hubbard model.49 They have been derived recently without refering explicitly to the spin-particle-hole coherent states, but by introducing a fluctuating spin-quantization axis in the functional integral 39 


\section{STRONG-COUPLING EXPANSION}

In this section, we recast the spin-particle-hole coherent-state path integral in a form suitable for a perturbative expansion with respect to $t / U$. This is achieved by performing two successive transformations of the intersite hopping term (Secs. III A and III B). The resulting action is expressed as a function of two fermionic fields $\left(\gamma_{\uparrow}\right.$ and $\left.\gamma_{\downarrow}\right)$ which describe the propagation of particles in the LHB and UHB, and a unit vector field $\boldsymbol{\Omega}$. In Sec. III C, we show that this action can be truncated to quartic order in the fermionic fields in the strong correlation limit. Finally, in Sec. IIID we show how this effective strong-coupling action can be used as the starting point of a diagrammatic perturbative expansion with respect to $t / U$, and discuss possible applications of our formalism.

\section{A. Grassmannian Hubbard-Stratonovich transformations}

Following Refs.40,25,39 we decouple the intersite hopping term in Eq. (2.34) by means of a Grassmannian Hubbard-Stratonovich transformation. The partition function becomes

$$
\begin{aligned}
Z= & \int \mathcal{D} \boldsymbol{\Omega} \operatorname{det}(\hat{t}) \int \mathcal{D}[\psi] \exp \left\{-\sum_{a, b} \psi_{a}^{*} \hat{t}_{a b}^{-1} \psi_{b}\right\} \\
& \times \int d \lambda \int \mathcal{D}[f, e, p, d] \exp \left\{-S_{\mathrm{at}}+\sum_{a}\left(\psi_{a}^{*} \gamma_{a}+\text { c.c. }\right)\right\}
\end{aligned}
$$

where $\psi$ is an auxiliary fermionic field which couples to the (composite) field $\gamma$ defined in Eq. (2.33). We use the notation

$$
\psi_{a} \equiv \psi_{\mathbf{r}_{a} \sigma_{a}}\left(\tau_{a}\right), \sum_{a} \equiv \sum_{\mathbf{r}_{a}, \sigma_{a}} \int d \tau_{a}
$$

and denote by

$$
\hat{t}_{\mathbf{r r}}=R_{\mathbf{r}}^{\dagger} t_{\mathbf{r r}^{\prime}} R_{\mathbf{r}^{\prime}}
$$

the $\boldsymbol{\Omega}$-dependent intersite hopping matrix. The $\mathrm{SU}(2) / \mathrm{U}(1)$ matrix $R_{\mathbf{r}}$ rotates the spin-quantization axis from $\hat{\mathbf{z}}$ to $\boldsymbol{\Omega}_{\mathbf{r}}$ [see Eq. (2.32)]. The diagonal elements of $\hat{t}_{\mathbf{r r}^{\prime}}$ correspond to intraband propagation, while its offdiagonal elements describe transitions between the LHB and UHB. Note that $\operatorname{det}(\hat{t})$ is a function of $\boldsymbol{\Omega}$ and should therefore be kept explicitly in the functional integral.

Performing the functional integral over the fields $f, e, p, d$ and the Lagrange multipliers $\lambda$, we obtain

$$
\begin{aligned}
\int d \lambda \int \mathcal{D}[f, e, p, d] \exp \left\{-S_{\text {at }}\right. & \left.+\sum_{a}\left(\psi_{a}^{*} \gamma_{a}+\text { c.c. }\right)\right\} \\
& =Z_{\text {at }}[\boldsymbol{\Omega}] e^{W\left[\psi^{*}, \psi ; \boldsymbol{\Omega}\right]}
\end{aligned}
$$

where

$$
\begin{aligned}
Z_{\text {at }}[\boldsymbol{\Omega}] & =\int d \lambda \int \mathcal{D}[f, e, p, d] e^{-S_{\mathrm{at}}} \\
& =Z_{\mathrm{at}}^{(0)} e^{-S_{B}[\boldsymbol{\Omega}]}
\end{aligned}
$$

is the "partition function" in the atomic limit $(t=0)$ for a given configuration of $\boldsymbol{\Omega} . Z_{\text {at }}^{(0)}$ is the partition function obtained from $S_{\text {at }}^{(0)}$. We emphasize here that the integration of the Lagrange multipliers and the bosonic fields has been done exactly. $S_{B}[\boldsymbol{\Omega}]$ is the action of the spin degrees of freedom in the atomic limit. Treating $A_{\mathbf{r}}^{0}=\left\langle\boldsymbol{\Omega}_{\mathbf{r}} \mid \dot{\boldsymbol{\Omega}}_{\mathbf{r}}\right\rangle$ in perturbation, we obtain to lowest order (in a cumulant expansion) 39

$$
\begin{aligned}
S_{B}[\boldsymbol{\Omega}] & =\sum_{\mathbf{r}} \int d \tau A_{\mathbf{r}}^{0}\left\langle f_{\mathbf{r} \uparrow}^{*} p_{\mathbf{r}}^{*} f_{\mathbf{r} \uparrow} p_{\mathbf{r}}\right\rangle_{S_{\mathrm{at}}^{(0)}} \\
& =\sum_{\mathbf{r}} \int d \tau A_{\mathbf{r}}^{0} .
\end{aligned}
$$

$S_{B}$ is a collection of Berry phase terms for spins localized at the lattice sites. In Eq. (3.4), $W\left[\psi^{*}, \psi ; \boldsymbol{\Omega}\right]$ is the generating functional of the connected atomic Green's functions

$$
\begin{aligned}
G_{\left\{a_{i}, b_{i}\right\}}^{R c} & =(-1)^{R}\left\langle\gamma_{a_{1}} \cdots \gamma_{a_{R}} \gamma_{b_{R}}^{*} \cdots \gamma_{b_{1}}^{*}\right\rangle_{\text {at }, \mathrm{c}} \\
& =\left.\frac{\delta^{(2 R)} W\left[\psi^{*}, \psi ; \boldsymbol{\Omega}\right]}{\delta \psi_{a_{1}}^{*} \cdots \delta \psi_{a_{R}}^{*} \delta \psi_{b_{R}} \cdots \delta \psi_{b_{1}}}\right|_{\psi^{*}=\psi=0} .
\end{aligned}
$$

Note that the $G^{R c}$ 's are defined for a given configuration of the field $\boldsymbol{\Omega}$.

The partition function is thus written as

$$
\begin{aligned}
& Z=Z_{\text {at }}^{(0)} \int \mathcal{D} \boldsymbol{\Omega} \operatorname{det}(\hat{t}) \int \mathcal{D}[\psi] e^{-S\left[\psi^{*}, \psi ; \boldsymbol{\Omega}\right]} \\
& S\left[\psi^{*}, \psi ; \boldsymbol{\Omega}\right]=S_{B}[\boldsymbol{\Omega}]+\sum_{a, b} \psi_{a}^{*} \hat{t}_{a b}^{-1} \psi_{b}-W\left[\psi^{*}, \psi ; \boldsymbol{\Omega}\right]
\end{aligned}
$$

$W\left[\psi^{*}, \psi ; \boldsymbol{\Omega}\right]$ can be obtained explicitly by inverting Eq. (3.7):

$$
W\left[\psi^{*}, \psi\right]=\sum_{R=1}^{\infty} \frac{(-1)^{R}}{(R !)^{2}} \sum_{a_{i}, b_{i}}^{\prime} \psi_{a_{1}}^{*} \cdots \psi_{a_{R}}^{*} \psi_{b_{R}} \cdots \psi_{b_{1}} G_{\left\{a_{i}, b_{i}\right\}}^{R c} .
$$

The primed summation in (3.9) reminds us that all the fields in a given product $\psi_{a_{1}}^{*} \cdots \psi_{b_{1}}$ share the same value of the site index.

In order to completely determine the action $S\left[\psi^{*}, \psi ; \boldsymbol{\Omega}\right]$, we need to compute the atomic Green's functions $G^{R c}$ from the action $S_{\text {at }}$. The procedure to obtain these quantities can be found in Appendix $\mathrm{C}$ and in Ref. 39. Here we only quote the final results. The single-particle Green's function is 


$$
G_{\mathbf{r} \sigma}^{-1}=G_{\sigma}^{(0)-1}-\operatorname{sgn}(\sigma) A_{\mathbf{r}}^{0},
$$

where $\operatorname{sgn}(\sigma)=1(-1)$ for $\sigma=\uparrow(\downarrow) . G^{(0)}$ is the atomic Green's function corresponding to $S_{\mathrm{at}}^{(0)}$. In the low temperature limit

$$
\begin{aligned}
& G_{\uparrow}^{(0)}(\tau)=\theta(-\tau+\eta) e^{\mu \tau}, \\
& G_{\downarrow}^{(0)}(\tau)=-\theta(\tau-\eta) e^{(\mu-U) \tau},
\end{aligned}
$$

where $\eta \rightarrow 0^{+}$. In Fourier space, Eqs. (3.11) become $G_{\uparrow}^{(0)}(i \omega)=(i \omega+\mu)^{-1}$ and $G_{\downarrow}^{(0)}(i \omega)=(i \omega+\mu-U)^{-1}$ where $\omega$ is a fermionic Matsubara frequency. As shown in Sec. (III d), the $R$-particle Green's functions $G^{R c}$ $(R \geq 2)$ are involved only in (virtual) interband transitions. The typical energy scale $(\sim U)$ for the latter is much larger than the typical energy scale for spin fluctuations $\left(\sim J=4 t^{2} / U\right)$ so that the effect of the Berry phase term $A^{0}$ can be neglected (i.e. we can use $S_{\text {at }}^{(0)}$ instead of $S_{\text {at }}$ to compute $G^{R c}$ for $R \geq 2$ ). We find

$$
G_{\sigma \cdots \sigma, \sigma \cdots \sigma}^{R c}=0(R \geq 2),
$$

which implies that there is no intraband interaction for the $\psi$ field. The two-particle atomic Green's function $G^{\mathrm{II} c}$ is thus entirely determined by $G_{\uparrow \downarrow \uparrow \downarrow}^{\mathrm{II} c}$, or equivalently by the two-particle vertex (see Eq. ( $\overline{\mathrm{C} 12})$ )

$$
\Gamma_{\uparrow \downarrow, \uparrow \downarrow}^{\mathrm{II}}\left(i \omega_{1}, i \omega_{2} ; i \omega_{3}\left(i \omega_{4}\right)\right)=i \omega_{2}-i \omega_{3}-U,
$$

where $\omega_{4}=\omega_{1}+\omega_{2}-\omega_{3}$ is fixed by energy conservation. We shall show in Sec. III C that the effective action in the strong-coupling regime $(t / U \ll 1)$ is entirely determined by the knowledge of $G$ and $G^{\mathrm{IIc} c}$ (or $\Gamma^{\mathrm{II}}$ ) so that we do not require the knowledge of $G^{R c}$ for $R \geq 3$.

We could now follow Ref. 25 and use the action $S\left[\psi^{*}, \psi ; \boldsymbol{\Omega}\right]$ to perform a diagrammatic perturbative expansion with respect to the intersite hopping amplitude $t$. The matrix structure of $\hat{t}_{\mathbf{r r}^{\prime}}$ distinguishes processes that do conserve the number of doubly occupied sites from those that involve interband transitions, thus allowing for a $t / U$ expansion. [In Ref. 25, the auxiliary field $\psi_{\sigma}$ couples to the original fermionic field $c_{\sigma}$. The resulting perturbative expansion involves two dimensionless parameters, $t / U$ and $t / T$, and breaks down at low temperature.] However, the drawback of using the action $S\left[\psi^{*}, \psi ; \boldsymbol{\Omega}\right]$ is that the field $\psi$ has no direct physical meaning. It is rather the fields to which $\psi_{\uparrow}$ and $\psi_{\downarrow}$ couple, namely the composite fields $e^{*} p f_{\uparrow}$ and $p^{*} d f_{\downarrow}$, which have a direct physical interpretation in terms of particles propagating in the LHB and UHB. This suggests to perform a second Hubbard-Stratonovich transformation of the intersite hopping term. Denoting by $\gamma$ the auxiliary field of this transformation, we rewrite the partition function as

$$
\begin{aligned}
Z= & Z_{\mathrm{at}}^{(0)} \int \mathcal{D} \boldsymbol{\Omega} \int \mathcal{D}[\gamma, \psi] \exp \left\{-S_{B}[\boldsymbol{\Omega}]+\sum_{a, b} \gamma_{a}^{*} \hat{t}_{a b} \gamma_{b}\right. \\
& \left.+\sum_{a}\left(\gamma_{a}^{*} \psi_{a}+\text { c.c. }\right)+W\left[\psi^{*}, \psi ; \boldsymbol{\Omega}\right]\right\} .
\end{aligned}
$$

Integrating out the $\psi$ field, we obtain

$$
\begin{aligned}
Z= & Z_{\mathrm{at}}^{(0)} \int \mathcal{D} \boldsymbol{\Omega} \tilde{Z}[\boldsymbol{\Omega}] e^{-S_{B}[\boldsymbol{\Omega}]}, \\
& \times \int \mathcal{D}[\gamma] \exp \left\{\sum_{a, b} \gamma_{a}^{*} \hat{t}_{a b} \gamma_{b}+\tilde{W}\left[\gamma^{*}, \gamma ; \boldsymbol{\Omega}\right]\right\},
\end{aligned}
$$

Note that $\gamma$ is now an elementary field. [We expect $\gamma$ to have the same physical meaning as the composite fields defined in Eq. (2.33), hence the same notation.] $\tilde{W}\left[\gamma^{*}, \gamma ; \boldsymbol{\Omega}\right]$ is the generating functional of the connected Green's functions $\tilde{G}^{R c}$ calculated with the action $-W\left[\psi^{*}, \psi ; \boldsymbol{\Omega}\right]$. Since the latter is local (in space), so are the $\tilde{G}^{R c}$ 's. $\tilde{Z}[\boldsymbol{\Omega}]$ is defined by

$$
\begin{aligned}
\tilde{Z}[\boldsymbol{\Omega}] & =\int \mathcal{D}[\psi] e^{W\left[\psi^{*}, \psi ; \boldsymbol{\Omega}\right]} \\
& =\operatorname{det}(-G) e^{-S^{\prime}[\boldsymbol{\Omega}]}
\end{aligned}
$$

$\operatorname{det}(-G)$ comes from the Gaussian part, $\sum_{a, b} G_{a b} \psi_{a}^{*} \psi_{b}$, of the action $-W\left[\psi^{*}, \psi ; \boldsymbol{\Omega}\right]$. Non-Gaussian terms give the contribution $S^{\prime}[\boldsymbol{\Omega}]$ :

$$
e^{-S^{\prime}[\boldsymbol{\Omega}]}=\left\langle\exp \left\{\sum_{R=2}^{\infty} \frac{(-1)^{R}}{(R !)^{2}} \sum_{a_{i}, b_{i}}^{\prime} \psi_{a_{1}}^{*} \cdots \psi_{b_{1}} G_{\left\{a_{i}, b_{i}\right\}}^{R c}\right\}\right\rangle
$$

where the average is to be taken with the action $\sum_{a, b} G_{a b} \psi_{a}^{*} \psi_{b} . \quad S^{\prime}[\boldsymbol{\Omega}]$ can be represented as a sum of Feynman diagrams, the bare propagator being $-G^{-1}$ and the vertices the atomic Green's functions $G^{R c}$ (with $R \geq 2$ ). An example of diagram is given in Fig. 2. [The symbols used in the Feynman diagrams are defined in Fig. 1.] This diagram, as well as the other diagrams contributing to $S^{\prime}[\boldsymbol{\Omega}]$ does not have any physical meaning. As will be shown below, $S^{\prime}[\boldsymbol{\Omega}]$ does not contribute to the final result. "Physical" and "anomalous" (i.e. without "physical" meaning) diagrams can be defined rigorously by considering the perturbation theory based on the action $S\left[\psi^{*}, \psi ; \boldsymbol{\Omega}\right]$ [Eq. (3.8)]. Within the latter, all diagrams can be represented by atomic Green's functions connected by the intersite hopping matrix $\hat{t}$. The perturbation theory based on the action $S\left[\gamma^{*}, \gamma ; \boldsymbol{\Omega}\right]$ generates diagrams that cannot be represented in this way. These "anomalous" diagrams should not contribute to physical quantities. Indeed, we will find that the total contribution of anomalous diagrams always vanishes (see Sec. III B). 


$$
\begin{aligned}
& G_{\sigma}=\longrightarrow \quad G_{\sigma}^{-1}= \\
& G^{R c}=\vdots \Gamma^{R}=\vdots \vdots \\
& \hat{t}=\ldots \ldots . \\
& \hat{\mathcal{G}}_{\uparrow}^{(0)}=\square
\end{aligned}
$$

FIG. 1. Definition of the various symbols appearing in the Feynman diagrams. Connected atomic Green's functions are represented by a filled circle and atomic vertices by polygons, with the exception of $G^{-1}$ which is also shown as a filled circle. The intersite hopping matrix $\hat{t}$ is represented by a dashed line, and the propagator $\hat{\mathcal{G}}_{\uparrow}^{(0)}$ defined in Eq. 3.37) by an empty circle.

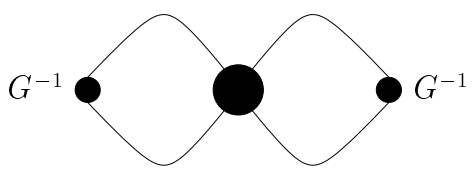

FIG. 2. A Feynman diagram contributing to $S^{\prime}[\boldsymbol{\Omega}]$.

Noting that $\operatorname{det}(-G)=Z_{\text {at }}^{-1}[\Omega], 50$ we obtain

$$
\begin{aligned}
Z & =\int \mathcal{D} \boldsymbol{\Omega} \int \mathcal{D}[\gamma] e^{-S\left[\gamma^{*}, \gamma ; \boldsymbol{\Omega}\right]} \\
S\left[\gamma^{*}, \gamma ; \boldsymbol{\Omega}\right] & =S^{\prime}[\boldsymbol{\Omega}]-\sum_{a, b} \gamma_{a}^{*} \hat{t}_{a b} \gamma_{b}-\tilde{W}\left[\gamma^{*}, \gamma ; \boldsymbol{\Omega}\right] .
\end{aligned}
$$

In order to completely determine the action $S\left[\gamma^{*}, \gamma ; \Omega\right]$, we need to compute the (local) Green's functions $\tilde{G}^{R c}$ from the action $-W\left[\psi^{*}, \psi ; \boldsymbol{\Omega}\right]$ [Eq. (3.9)].

Let us first consider the single-particle Green's function $\tilde{G}_{a b}=-\left\langle\psi_{a} \psi_{b}^{*}\right\rangle_{-W}$. Retaining only the Gaussian part of the action $-W$, i.e. $\sum_{a, b} G_{a b} \psi_{a}^{*} \psi_{b}$, one obtains $\tilde{G}=-G^{-1}$. More generally, we have

$$
\tilde{G}_{a b}=-G_{a b}^{-1}+\tilde{\Gamma}_{a b}
$$

where $\tilde{\Gamma}$ can be represented as a sum of Feynman diagrams arising from the vertices $G^{R c}$ (with $R \geq 2$ ). An example is shown in Fig. 3. It is easy to see that all the diagrams contributing to $\tilde{\Gamma}$ are anomalous. The fact that $\tilde{\Gamma}$ has no physical meaning can be understood as follows. We can view $\tilde{\Gamma}$ as a self-energy correction to $G$ [see Eq. (3.19)]. Since $G$ is the exact atomic Green's function, we do not expect any local (i.e. atomic) correction. We will see in Sec. III B that the role of $\tilde{\Gamma}$ is to cancel other anomalous contributions arising in the strong-coupling perturbative expansion.

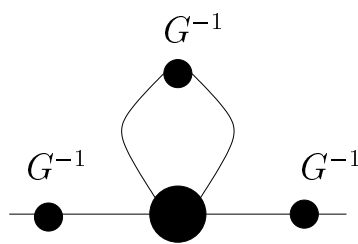

FIG. 3. A Feynman diagram contributing to $\tilde{\Gamma}$.

A result similar to Eq. (3.19) holds for higherorder Green's functions. The simplest diagram for the two-particle Green's function $\tilde{G}_{a_{1} a_{2}, b_{1} b_{2}}^{\mathrm{II} c}=$ $\left\langle\psi_{a_{1}} \psi_{a_{2}} \psi_{b_{2}}^{*} \psi_{b_{1}}^{*}\right\rangle_{-W}$ (shown in Fig. Wa) gives the contribution $-\Gamma_{a_{1} a_{2}, b_{1} b_{2}}^{\mathrm{II}}$, where $\Gamma_{a_{1} a_{2}, b_{1} b_{2}}^{\mathrm{II}}$ is the two-particle vertex. All other diagrams are anomalous (Fig. $1 \mathrm{~b}$ ). In the same way, $\tilde{G}^{\text {IIIc }}$ can be written as the sum of $\Gamma^{\mathrm{III}}$ and

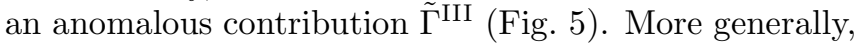
we find

$$
\tilde{G}_{\left\{a_{i}, b_{i}\right\}}^{R c}=-(-1)^{R}\left(\Gamma_{\left\{a_{i}, b_{i}\right\}}^{R}+\tilde{\Gamma}_{\left\{a_{i}, b_{i}\right\}}^{R}\right)(R \geq 2),
$$

where $\Gamma^{R}$ is the atomic $R$-particle vertex and $\tilde{\Gamma}^{R}$ denotes anomalous contributions.

(a)

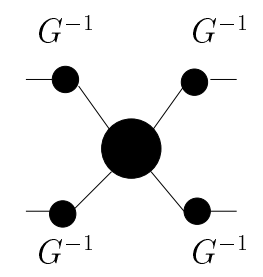

(b)

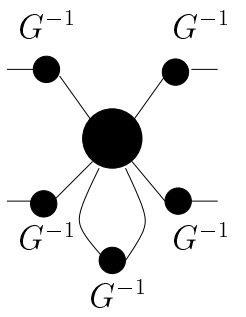

FIG. 4. Feynman diagrams for $\tilde{G}^{\mathrm{II} c}$. (a) $-\Gamma^{\mathrm{II}}$. (b) Anomalous diagram. 
(a)

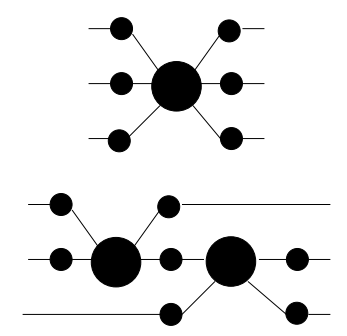

(b)

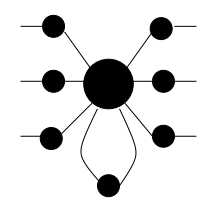

FIG. 5. Examples of Feynman diagrams contributing to $\tilde{G}^{\mathrm{III} c}$. (a) Contributions to $\Gamma^{\mathrm{III}}$. (b) A contribution to the anomalous part $\tilde{\Gamma}^{\mathrm{III}}$. All the filled circles with two external legs denote $G^{-1}$.

We can then rewrite the action as

$$
\begin{aligned}
& S\left[\gamma^{*}, \gamma ; \boldsymbol{\Omega}\right]=S^{\prime}[\boldsymbol{\Omega}]-\sum_{a, b} \gamma_{a}^{*}\left(\hat{t}_{a b}+G_{a b}^{-1}-\tilde{\Gamma}_{a b}\right) \gamma_{b} \\
& +\sum_{R=2}^{\infty} \frac{1}{(R !)^{2}} \sum_{a_{i}, b_{i}}^{\prime}\left(\Gamma_{\left\{a_{i}, b_{i}\right\}}^{R}+\tilde{\Gamma}_{\left\{a_{i}, b_{i}\right\}}^{R}\right) \gamma_{a_{1}}^{*} \cdots \gamma_{b_{1}} .
\end{aligned}
$$

Note that this result has been obtained without any approximation and gives the exact value of the partition function if one carries out the functional integral over the fields $\gamma_{\sigma}$ and $\boldsymbol{\Omega}$. By performing two successive Hubbard-Stratonovich transformations, we have summed the atomic contributions to the single-particle propagator and the $R$-particle vertices $(R \geq 2)$. As a result, the action $S\left[\gamma^{*}, \gamma ; \boldsymbol{\Omega}\right]$ is essentially parametrized by the intersite hopping matrix $\hat{t}$ and the atomic vertices $G^{-1}$ and $\Gamma^{R}(R \geq 2)$. [We show in Sec. III B how to deal with the anomalous vertices $\tilde{\Gamma}^{R}$.] Using $S\left[\gamma^{*}, \gamma ; \boldsymbol{\Omega}\right]$ instead of $S[f, e, p, d, \lambda ; \boldsymbol{\Omega}]$ [Eq. (2.34)] or $S\left[\psi^{*}, \psi ; \boldsymbol{\Omega}\right]$ [Eq. (3.8)] presents two main advantages. First we now deal with only three fields whose physical meaning is clear. $\gamma_{\uparrow}$ and $\gamma_{\downarrow}$ describe particles propagating in the LHB and UHB, respectively, while the dynamics of $\boldsymbol{\Omega}$ arises from spin fluctuations. [Note however that the Green's function of the original fermions (those involved in the definition of the Hubbard model) is not merely the propagator $-\left\langle\gamma_{a} \gamma_{b}^{*}\right\rangle$ (see Sec. [IID).] Second, $S\left[\gamma^{*}, \gamma ; \boldsymbol{\Omega}\right]$ turns out to be a convenient starting point in the strong correlation limit $t / U \ll 1$. Indeed, for $U \rightarrow \infty$ (no interband transition), only the Gaussian part of the action needs to be considered. The lowest-order corrections in $t / U$ are determined by a small number of atomic vertices. In practice, $S\left[\gamma^{*}, \gamma ; \boldsymbol{\Omega}\right]$ can be truncated to quartic order in the fermionic fields and is then parametrized only by $G^{-1}$ and $\Gamma^{\mathrm{II}}$ (Sec. III C).

\section{B. Anomalous diagrams}

In order to understand the role of anomalous diagrams, it is useful to first consider the atomic limit $(t=0)$ of the action (3.21). In that limit, one has to recover the known (exact) results.

Let us consider first the partition function. Integrating out the $\gamma$ field, one obtains

$$
\begin{aligned}
Z_{\mathrm{at}}[\boldsymbol{\Omega}]= & \operatorname{det}\left(-G^{-1}\right) e^{-S^{\prime}[\boldsymbol{\Omega}]-S^{\prime \prime}[\boldsymbol{\Omega}]}, \\
e^{-S^{\prime \prime}[\boldsymbol{\Omega}]}= & \left\langle\operatorname { e x p } \left\{-\sum_{a, b} \gamma_{a}^{*} \tilde{\Gamma}_{a b} \gamma_{b}\right.\right. \\
& \left.\left.-\sum_{R=2}^{\infty} \frac{1}{(R !)^{2}} \sum_{a_{i}, b_{i}}^{\prime}\left(\Gamma_{\left\{a_{i}, b_{i}\right\}}^{R}+\tilde{\Gamma}_{\left\{a_{i}, b_{i}\right\}}^{R}\right) \gamma_{a_{1}}^{*} \cdots \gamma_{b_{1}}\right\}\right\rangle
\end{aligned}
$$

where the average is to be taken with the Gaussian action $-\sum_{a, b} \gamma_{a}^{*} G_{a b}^{-1} \gamma_{b}$. In order to obtain the correct result

$$
\begin{aligned}
Z_{\mathrm{at}} & =\int \mathcal{D} \boldsymbol{\Omega} \operatorname{det}\left(-G^{-1}\right) \\
& =Z_{\mathrm{at}}^{(0)} \int \mathcal{D} \boldsymbol{\Omega} e^{-S_{B}[\boldsymbol{\Omega}]},
\end{aligned}
$$

one must have $S^{\prime}[\boldsymbol{\Omega}]+S^{\prime \prime}[\boldsymbol{\Omega}]=0$. This result can be obtained by inspection. Consider for instance the diagrams containing once and only once the vertex $\Gamma^{\mathrm{II}}$ (and no higher-order vertex). The diagram contributing to $S^{\prime}[\boldsymbol{\Omega}]$ is shown in Fig. 2 and has the overall factor $-1 / 2$. There are two contributions to $S^{\prime \prime}[\Omega]$. They come from the terms $\left\langle\gamma_{a}^{*} \tilde{\Gamma}_{a b} \gamma_{b}\right\rangle$ and $\left\langle\Gamma_{a_{1} a_{2}, b_{1} b_{2}}^{\mathrm{II}} \gamma_{a_{1}}^{*} \gamma_{a_{2}}^{*} \gamma_{b_{2}} \gamma_{b_{1}}\right\rangle$ where the average is taken with the action $-\sum_{a, b} \gamma_{a}^{*} G_{a b}^{-1} \gamma_{b}$ [see Eq. (3.22)]. Both contributions correspond to the diagram of Fig. 2, but with the factors 1 and $-1 / 2$. We conclude that the total contribution to $S^{\prime}+S^{\prime \prime}$ vanishes.

In the same way, we can calculate the single-particle Green's function $-\left\langle\gamma_{a} \gamma_{b}^{*}\right\rangle$ (for a given configuration of $\boldsymbol{\Omega})$ and verify that we do obtain the correct result in the atomic limit, namely $G_{a b}$. From the action (3.21), we read off the inverse (atomic) propagator $G^{-1}-\tilde{\Gamma}$. Selfenergy corrections due to vertices $R \geq 2$ should therefore cancel $\tilde{\Gamma}$. Again this result can be proved by inspection. Consider again the diagrams containing once the vertex $\Gamma^{\mathrm{II}}$. The contribution to $\tilde{\Gamma}$ is shown in Fig. 3 . The one-loop self-energy correction coming from $\Gamma^{\mathrm{II}}$ (see Eq. (3.21)) produces the same diagram but with opposite sign.

Thus we come to the conclusion that anomalous diagrams cancel in the atomic limit. We expect this property to hold also when $t \neq 0$, since anomalous diagrams do not have any physical meaning. Consider for instance the anomalous diagrams shown in Fig. 6 a which contribute to the effective action of the spin degrees of free$\operatorname{dom} S[\boldsymbol{\Omega}]=-\ln Z[\boldsymbol{\Omega}]$. [Fig. $6 \mathrm{~b}$ shows the corresponding "physical" diagram.] Working out signs and symmetry factors, we find that the sum of the two diagrams of 
Fig. 6 a vanishes if $\tilde{\Gamma}$ is approximated by the contribution shown diagrammatically in Fig. 3 .

(a)

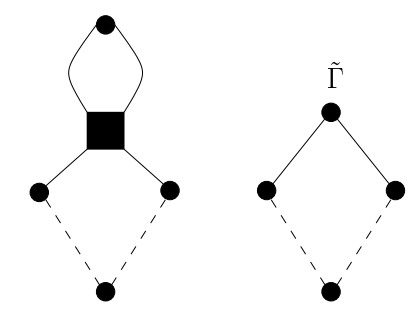

(b)

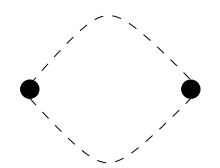

FIG. 6. (a) Two anomalous diagrams for the free energy. Their sum vanishes when $\tilde{\Gamma}$ is approximated by the contribution shown diagrammatically in Fig. 3. (b) The corresponding "physical" diagram.

As expected on physical grounds, anomalous diagrams always cancel in the calculation of physical quantities. We can then work with the action

$$
\begin{aligned}
S\left[\gamma^{*}, \gamma ; \boldsymbol{\Omega}\right]= & -\sum_{a, b} \gamma_{a}^{*}\left(\hat{t}_{a b}+G_{a b}^{-1}\right) \gamma_{b} \\
& +\sum_{R=2}^{\infty} \frac{1}{(R !)^{2}} \sum_{a_{i}, b_{i}}^{\prime} \Gamma_{\left\{a_{i}, b_{i}\right\}}^{R} \gamma_{a_{1}}^{*} \cdots \gamma_{b_{1}}
\end{aligned}
$$

provided that we discard all anomalous diagrams.

\section{Effective action of the Hubbard model in the strong correlation limit}

In this section, we show that only the quadratic and quartic parts of the action $S\left[\gamma^{*}, \gamma ; \boldsymbol{\Omega}\right]$ need to be considered in the strong-coupling limit. This is achieved by considering the effective action $S_{\mathrm{LHB}}\left[\gamma_{\uparrow}^{*}, \gamma_{\uparrow} ; \boldsymbol{\Omega}\right]$ of particles propagating in the LHB. To first order in $t / U$, $S_{\mathrm{LHB}}\left[\gamma_{\uparrow}^{*}, \gamma_{\uparrow} ; \boldsymbol{\Omega}\right]$ should correspond to the $t$ - $J$ model. This result will guide us in the determination of the leading terms in the action of the Hubbard model in the strong correlation limit. We emphasize however that our primary goal is not to determine the effective action of carriers in the LHB, but to derive an effective action for the full Hubbard model in the strong correlation limit. As discussed at the end of this section, there are advantages in working with the Hubbard model instead of considering the $t-J$ model obtained by integrating out the LHB.

$S_{\mathrm{LHB}}$ is obtained by integrating out the $\gamma_{\downarrow}$ field: 39

$$
e^{-S_{\mathrm{LHB}}\left[\gamma_{\uparrow}^{*}, \gamma_{\uparrow} ; \boldsymbol{\Omega}\right]}=\int \mathcal{D}\left[\gamma_{\downarrow}\right] e^{-S\left[\gamma^{*}, \gamma ; \boldsymbol{\Omega}\right]} .
$$

At zeroth order in $t / U$, interband transitions are neglected. Since $\Gamma_{\sigma \cdots \sigma}^{R}=0$, diagrams contributing to $S_{\mathrm{LHB}}^{(0)}$ must necessary contain closed loops of $\gamma_{\downarrow}$ particle. In the absence of particles in the UHB, these loops vanish (see Appendix (D). As a result, the effective action of the LHB is Gaussian to leading order in $t / U$ :

$$
\begin{aligned}
S_{\mathrm{LHB}}^{(0)}= & \sum_{\mathbf{r}} \int d \tau \gamma_{\mathbf{r} \uparrow}^{*}\left(\partial_{\tau}-\mu+A_{\mathbf{r}}^{0}\right) \gamma_{\mathbf{r} \uparrow} \\
& -\sum_{\mathbf{r}, \mathbf{r}^{\prime}} \int d \tau \gamma_{\mathbf{r} \uparrow}^{*} \hat{t}_{\mathbf{r} \uparrow, \mathbf{r}^{\prime} \uparrow} \gamma_{\mathbf{r}^{\prime} \uparrow} .
\end{aligned}
$$

The action (3.26) describes particles propagating in the LHB and interacting with spin fluctuations via the gauge field $A^{0}$ and the $\boldsymbol{\Omega}$-dependent intersite hopping matrix $\hat{t}$ (i.e. $S_{\mathrm{LHB}}^{(0)}$ describes fermions coupled to a $\mathrm{U}(1)$ gauge field). A detailed discussion of its physical meaning can be found in Sec. III.B.1 of Ref. 39 .

We now consider the contributions of order $t / U$ to $S_{\text {LHB }}$ [Eq. (3.25)]. They are represented by the diagrams of Fig. 7. Each of these diagrams contains two interband transitions. The vanishing of closed loops with $\gamma_{\downarrow}$ particles ensures that all the diagrams to be considered are of the type shown in Fig. Ja. Examples of vanishing diagrams are shown in Fig. $7 \mathrm{~b}$.

(a)
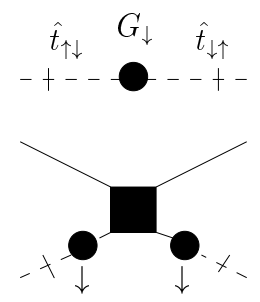

(b)

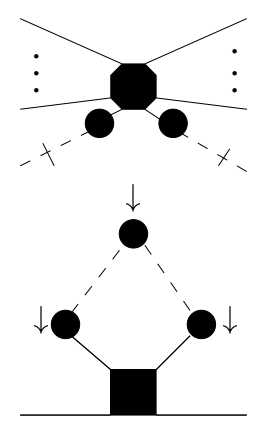

FIG. 7. (a) Contributions of order $t / U$ to the effective action $S_{\text {LHB }}$. (b) An example of vanishing diagram. Slashed dashed lines indicate interband transitions.

Let us first focus on the quadratic and quartic contributions generated by the integration of the UHB (first two diagrams of Fig. 7 a). These terms are non-local in time. However, since they involve (virtual) interband transitions, they can be approximated by local vertices. From the equations of motion in the atomic limit, we deduce $\gamma_{\uparrow}\left(\tau^{\prime}\right)=e^{-\mu\left(\tau-\tau^{\prime}\right)} \gamma_{\uparrow}(\tau)$.51 Using the latter equation and integrating over the time differences, the non-local 
vertices are then approximated by local vertices. For instance, the two-point vertex (first diagram of Fig. $7 \mathrm{a}$ ) is approximated as

$$
\begin{aligned}
& \sum_{\mathbf{r}, \mathbf{r}^{\prime}, \mathbf{r}^{\prime \prime}} \int d \tau d \tau^{\prime} \gamma_{\mathbf{r} \uparrow}^{*}(\tau) \hat{t}_{\mathbf{r} \uparrow, \mathbf{r}^{\prime} \downarrow}(\tau) G_{\downarrow}^{(0)}\left(\tau-\tau^{\prime}\right) \hat{t}_{\mathbf{r}^{\prime} \downarrow, \mathbf{r}^{\prime \prime} \uparrow}\left(\tau^{\prime}\right) \gamma_{\mathbf{r}^{\prime \prime} \uparrow}\left(\tau^{\prime}\right) \\
& \simeq-\frac{1}{U} \sum_{\mathbf{r}, \mathbf{r}^{\prime}, \mathbf{r}^{\prime \prime}} \int d \tau \gamma_{\mathbf{r} \uparrow}^{*}(\tau) \hat{t}_{\mathbf{r} \uparrow, \mathbf{r}^{\prime} \downarrow}(\tau) t_{\mathbf{r}^{\prime} \downarrow, \mathbf{r}^{\prime \prime} \uparrow}(\tau) \gamma_{\mathbf{r}^{\prime \prime} \uparrow}(\tau),
\end{aligned}
$$

where we have used Eq. 3.11). Since we can ignore the effect of spin fluctuations on (virtual) interband transitions, we have replaced $G_{\downarrow}$ by $G_{\downarrow}^{(0)}$. If we also approximate the quartic term by a local vertex and neglect higher-order vertices, we end up with an effective action which is nothing but the action of the $t-J$ model (including the so-called pair-hopping terms) in the spinhole coherent-state path integral. 52 [See Ref. 39 for a detailed derivation.] It is crucial here that the effective action is local in time, otherwise it would not derive from an Hamiltonian. It should be noted that a similar approximation is made in the standard derivation of the $t-J$ model, which starts from the resolvant operator $\hat{R}(E)=(E-\hat{H})^{-1}$. Projecting out states with double occupancy yields an effective "Hamiltonian" $\hat{H}_{\text {eff }}(E)$ which depends on the energy $E$. The Hamiltonian of the $t-J$ model is obtained by retaining terms of order $O(t / U)$ and replacing $E_{\text {by }}$ the energy $E_{0}$ in the absence of interband coupling 43,53 In our formalism, this last step amounts to approximating the vertices of $S_{\mathrm{LHB}}$ by local vertices (in time) using the atomic equations of motion for the $\gamma_{\uparrow}$ field.

Since the quadratic and quartic terms of the action $S_{\text {LHB }}$ are sufficient to recover the action of the $t$ - $J$ model, we expect higher-order vertices to vanish at the same level of approximation. Consider the contribution of order $t / U$ to $S_{\text {LHB }}$ due to $\Gamma_{\uparrow \uparrow \downarrow, \uparrow \uparrow \downarrow}^{\text {III }}$ (Fig. 可a). This term involves the product

$$
\gamma_{\mathbf{r} \uparrow}^{*}\left(\tau_{1}\right) \gamma_{\mathbf{r} \uparrow}^{*}\left(\tau_{2}\right) \gamma_{\mathbf{r}^{\prime} \uparrow}^{*}\left(\tau_{3}\right) \gamma_{\mathbf{r}^{\prime \prime} \uparrow}\left(\tau_{3}^{\prime}\right) \gamma_{\mathbf{r} \uparrow}\left(\tau_{2}^{\prime}\right) \gamma_{\mathbf{r} \uparrow}\left(\tau_{1}^{\prime}\right)
$$

where the fields are evaluated at different times and three different sites $\left(\mathbf{r}, \mathbf{r}^{\prime}\right.$ and $\left.\mathbf{r}^{\prime \prime}\right)$. If we now approximate this term by using the atomic equation of motion, we obtain the product $\left[\gamma_{\mathbf{r} \uparrow}^{*}(\tau)\right]^{2} \gamma_{\mathbf{r}^{\prime} \uparrow}^{*}(\tau) \gamma_{\mathbf{r}^{\prime \prime} \uparrow}(\tau)\left[\gamma_{\mathbf{r} \uparrow}(\tau)\right]^{2}$ which vanishes since it involves squares of Grassmann variables. The same reasoning holds for higher-order vertices. Therefore, using the same approximations that lead to the $t-J$ model, only the quadratic and quartic terms of the action $S_{\text {LHB }}$ subsist.

We conclude that in the strong-coupling limit, we can truncate the action (3.24) to quartic order in the fermionic fields:

$$
\begin{aligned}
S\left[\gamma^{*}, \gamma ; \boldsymbol{\Omega}\right]= & \sum_{\mathbf{r}} \int d \tau \gamma_{\mathbf{r} \uparrow}^{*}\left(\partial_{\tau}-\mu+A_{\mathbf{r}}^{0}\right) \gamma_{\mathbf{r} \uparrow}-\sum_{\mathbf{r}, \mathbf{r}^{\prime}} \int d \tau \gamma_{\mathbf{r} \uparrow}^{*} \hat{t}_{\mathbf{r} \uparrow, \mathbf{r}^{\prime} \uparrow} \gamma_{\mathbf{r}^{\prime} \uparrow} \\
& +\sum_{\mathbf{r}} \int d \tau \gamma_{\mathbf{r} \downarrow}^{*}\left(\partial_{\tau}-\mu+U-A_{\mathbf{r}}^{0}\right) \gamma_{\mathbf{r} \downarrow}-\sum_{\mathbf{r}, \mathbf{r}^{\prime}} \int d \tau \gamma_{\mathbf{r} \downarrow}^{*} \hat{t}_{\mathbf{r} \downarrow, \mathbf{r}^{\prime} \downarrow} \gamma_{\mathbf{r}^{\prime} \downarrow} \\
& -\sum_{\mathbf{r}, \mathbf{r}^{\prime}} \int d \tau\left(\gamma_{\mathbf{r} \uparrow}^{*} \hat{t}_{\mathbf{r} \uparrow, \mathbf{r}^{\prime} \downarrow} \gamma_{\mathbf{r}^{\prime} \downarrow}+\text { c.c. }\right) \\
& +\sum_{\mathbf{r}} \int d \tau_{1} d \tau_{2} d \tau_{3} d \tau_{4} \Gamma_{\uparrow \downarrow, \uparrow \downarrow}^{\mathrm{II}}\left(\tau_{1}, \tau_{2} ; \tau_{3}, \tau_{4}\right) \gamma_{\mathbf{r} \uparrow}^{*}\left(\tau_{1}\right) \gamma_{\mathbf{r} \downarrow}^{*}\left(\tau_{2}\right) \gamma_{\mathbf{r} \downarrow}\left(\tau_{4}\right) \gamma_{\mathbf{r} \uparrow}\left(\tau_{3}\right) .
\end{aligned}
$$

Eq. (3.29) is the main result of this paper. It gives the effective action of the Hubbard model in the strongcoupling limit $t / U \ll 1$. The fields $\gamma_{\uparrow}$ and $\gamma_{\downarrow}$ correspond to particles propagating in the LHB and the UHB. They are coupled by the interband hopping matrix $\hat{t}_{\uparrow \downarrow}$ and the two-particle atomic vertex $\Gamma_{\uparrow \downarrow, \uparrow \downarrow}^{\mathrm{II}}$, and interact with the spin fluctuations which show up in the dynamics of $\boldsymbol{\Omega}$. Note that one can describe the interaction with spin fluctuations by a $\mathrm{SU}(2)$ gauge field with $A^{0}$ its time component.

The action (3.29) is suitable for a perturbative calculation of the free energy and the LHB Green's functions. However, except at half-filling, it does not allow to compute the UHB Green's functions. In a hole doped system, closed loops of $\gamma_{\uparrow}$ particles do not vanish (see Appendix D) so that the arguments used above for the LHB do not hold for the UHB. [Even at zeroth order in $t / U$, the effec- tive action $S_{\text {UHB }}$ contains an infinite number of terms.]

An interesting aspect of this action is that the strong local Coulomb repulsion is already present in the quadratic part of the action. To understand the physical meaning of $\Gamma_{\uparrow \downarrow, \uparrow \downarrow}^{\mathrm{II}}$, we rewrite Eq. (3.13) for real frequen$\operatorname{cies}(i \omega \rightarrow \omega)$ as

$$
E_{\mathrm{UHB}}-E_{\mathrm{LHB}}=U+\Gamma_{\uparrow \downarrow, \uparrow \downarrow}^{\mathrm{II}},
$$

where we have identified $\omega_{2}\left(\omega_{3}\right)$ with the energy of the particle in the UHB (LHB). Eq. (3.30) suggests that we can interpret $\Gamma_{\uparrow \downarrow, \uparrow \downarrow}^{\mathrm{II}}$ as the residual interaction (given that a part of the interaction is included in the quadratic action) between two particles siting at the same site. Alternatively, one can view the $U$ term in the action as a mean field, and $\Gamma_{\uparrow \downarrow, \uparrow \downarrow}^{\mathrm{II}}$ as the correction to this mean field.

Finally, we note that the action (3.29) without the quartic term is similar to that obtained within the 
large- $U$ Hartree-Fock approximation where the $\mathrm{SU}(2)$ spin-rotation invariance is maintained by introducing a fluctuating spin-quantization axis $\boldsymbol{\Omega}$ in the functional integral. 35 Omitting the quartic term $\left(\Gamma_{\uparrow \downarrow, \uparrow \downarrow}^{\mathrm{II}}\right)$ is however in general not possible without missing processes of order $t / U$. For instance, this termplays a central role in the derivation of the $t-J$ model 39

\section{Strong-coupling diagrammatic perturbation theory}

The action (3.29) can be used as the starting point for a perturbative calculation with respect to $t / U$. We discuss in the following the computation of the free energy and the LHB Green's function to first order in $t / U$. Formally, we have

$$
\begin{aligned}
Z & =\int \mathcal{D} \boldsymbol{\Omega} Z[\boldsymbol{\Omega}], \\
Z[\boldsymbol{\Omega}] & =e^{-S[\boldsymbol{\Omega}]}=\int \mathcal{D}[\gamma] e^{-S\left[\gamma^{*}, \gamma ; \boldsymbol{\Omega}\right]},
\end{aligned}
$$

and

$$
\begin{aligned}
\mathcal{G}_{\sigma}^{\mathrm{LHB}}(a, b) & =\frac{1}{Z} \int \mathcal{D} \boldsymbol{\Omega} e^{-S[\boldsymbol{\Omega}]}\left(R_{a}\right)_{\sigma \uparrow} \hat{\mathcal{G}}_{\uparrow}(a, b)\left(R_{b}^{\dagger}\right)_{\uparrow \sigma} \\
\hat{\mathcal{G}}_{\uparrow}(a, b) & =-\frac{1}{Z[\boldsymbol{\Omega}]} \int \mathcal{D}[\gamma] \gamma_{a \uparrow} \gamma_{b \uparrow}^{*} e^{-S\left[\gamma^{*}, \gamma ; \boldsymbol{\Omega}\right]}
\end{aligned}
$$

where $S[\boldsymbol{\Omega}]$ is the action for the spin degrees of freedom and $\hat{\mathcal{G}}_{\uparrow}(a, b)$ the Green's function for a given configuration of $\boldsymbol{\Omega}$. Note that the Green's function of the original fermions $c$ (those involved in the definition of the Hubbard model) is not merely $-\left\langle\gamma_{a} \gamma_{b}^{*}\right\rangle$ (see Appendix E). Eq. (3.32) shows that the fermionic field $c^{\text {LHB }}$ in the LHB can be expressed as

$$
\begin{aligned}
c_{\mathbf{r} \sigma}^{\mathrm{LHB}} & =\left(R_{\mathbf{r}}\right)_{\sigma \uparrow} \gamma_{\mathbf{r} \uparrow} \\
& =z_{\mathbf{r} \sigma} \gamma_{\mathbf{r} \uparrow},
\end{aligned}
$$

where the last line is obtained by writing the $\mathrm{SU}(2) / \mathrm{U}(1)$ rotation matrix $R_{\mathbf{r}}$ as

$$
R_{\mathbf{r}}=\left(\begin{array}{cc}
z_{\mathbf{r} \uparrow} & -z_{\mathbf{r} \downarrow}^{*} \\
z_{\mathbf{r} \downarrow} & z_{\mathbf{r} \uparrow}^{*}
\end{array}\right),
$$

with the constraint $\left|z_{\mathbf{r} \uparrow}^{2}\right|+\left|z_{\mathbf{r} \downarrow}^{2}\right|=1$. Eq. (3.35) is familiar from the Schwinger-boson slave-fermion formulation of the $t-J$ model 54

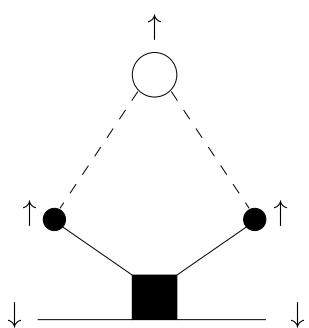

FIG. 8. An example of self-energy diagram for the UHB particles which is $O(1)$ in $t / U$.

We now introduce the propagator

$$
\hat{\mathcal{G}}_{\sigma}^{(0)-1}=G_{\sigma}^{-1}+\hat{t}_{\sigma \sigma}
$$

To zeroth order in $t / U$, the LHB Green's function is $\hat{\mathcal{G}}_{\uparrow}^{(0)}$, and the partition function is given by

$$
Z^{(0)}[\boldsymbol{\Omega}]=\operatorname{det}\left(-\hat{\mathcal{G}}_{\uparrow}^{(0)-1}\right)
$$

As discussed in Sec. III C $\hat{\mathcal{G}}_{\downarrow}^{(0)}$ is not the UHB Green's function to zeroth order in $t / U$ except at half-filling. Even in the absence of interband transition, there are $O(1)$ corrections to $\hat{\mathcal{G}}_{\downarrow}^{(0)}$ due to the non-vanishing of closed loops of $\gamma_{\uparrow}$ particles in the (hole) doped system (see Appendix D). A finite $O(1)$ self-energy correction to $\hat{\mathcal{G}}_{\downarrow}^{(0)}$ is shown in Fig. 8 .

(a)

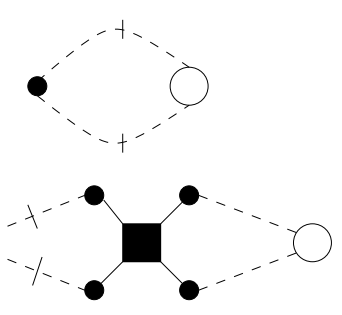

(c)

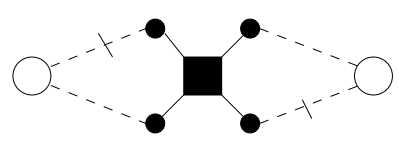

FIG. 9. Diagrammatic representation of the effective action of the spin degrees of freedom at order $O(t / U)$. (a) $S_{1}[\boldsymbol{\Omega}]$. (b) $S_{1}^{\prime}[\boldsymbol{\Omega}]$. (c) $S_{1}^{\prime \prime}[\boldsymbol{\Omega}]$. Slashed dashed lines indicate interband transitions.

To first order in $t / U$, there are three contributions to $S[\boldsymbol{\Omega}]$ shown diagrammatically in Fig. 9:

$$
\begin{aligned}
S_{1}[\boldsymbol{\Omega}]= & \sum_{a, b, a^{\prime}, b^{\prime}} \hat{\mathcal{G}}_{\uparrow}^{(0)}(a, b) \hat{t}_{\uparrow \downarrow}\left(b, b^{\prime}\right) G_{\downarrow}\left(b^{\prime}, a^{\prime}\right) \hat{t}_{\downarrow \uparrow}\left(a, a^{\prime}\right), \\
S_{1}^{\prime}[\boldsymbol{\Omega}]= & \sum_{a, b, a^{\prime}, b^{\prime}} \sum_{a_{i}, b_{i}} \Gamma_{\uparrow \downarrow, \uparrow \downarrow}^{\mathrm{II}}\left(a, b ; a^{\prime}, b^{\prime}\right) G_{\uparrow}\left(a^{\prime}, a_{4}\right) \hat{t}_{\uparrow \uparrow}\left(a_{4}, a_{3}\right) \hat{\mathcal{G}}_{\uparrow}^{(0)}\left(a_{3}, a_{2}\right) \hat{t}_{\uparrow \uparrow}\left(a_{2}, a_{1}\right) G_{\uparrow}\left(a_{1}, a\right) \\
& \times G_{\downarrow}\left(b^{\prime}, b_{4}\right) \hat{t}_{\downarrow \uparrow}\left(b_{4}, b_{3}\right) \hat{\mathcal{G}}_{\uparrow}^{(0)}\left(b_{3}, b_{2}\right) \hat{t}_{\uparrow \downarrow}\left(b_{2}, b_{1}\right) G_{\downarrow}\left(b_{1}, b\right),
\end{aligned}
$$




$$
\begin{aligned}
S_{1}^{\prime \prime}[\boldsymbol{\Omega}]= & -\sum_{a, b, a^{\prime}, b^{\prime}} \sum_{a_{i}, b_{i}} \Gamma_{\uparrow \downarrow, \uparrow \downarrow}^{\mathrm{II}}\left(a, b ; b^{\prime}, a^{\prime}\right) G_{\uparrow}\left(b^{\prime}, b_{4}\right) \hat{t}_{\uparrow \uparrow}\left(b_{4}, b_{3}\right) \hat{\mathcal{G}}_{\uparrow}^{(0)}\left(b_{3}, b_{2}\right) \hat{t}_{\uparrow \downarrow}\left(b_{2}, b_{1}\right) G_{\downarrow}\left(b_{1}, b\right) \\
& \times G_{\downarrow}\left(a^{\prime}, a_{4}\right) \hat{t}_{\downarrow \uparrow}\left(a_{4}, a_{3}\right) \hat{\mathcal{G}}_{\uparrow}^{(0)}\left(a_{3}, a_{2}\right) \hat{t}_{\uparrow \uparrow}\left(a_{2}, a_{1}\right) G_{\uparrow}\left(a_{1}, a\right) .
\end{aligned}
$$

The effective action $S[\Omega]$ of the spin degrees of freedom to order $O(t / U)$ can be computed exactly at halffilling. The zeroth-order contribution [Eq. (3.38)] yields the Berry phase term $S_{B}$ to lowest order in $A^{0}$. When computing $S_{1}, S_{1}^{\prime}$ and $S_{1}^{\prime \prime}$ we may replace $\hat{\mathcal{G}}_{\uparrow}^{(0)}$ by $G_{\uparrow}$ since the neglected terms will be of higher-order in $t / U .5$ Furthermore, since $S_{1}, S_{1}^{\prime}$ and $S_{1}^{\prime \prime}$ describe (virtual) interband transitions, we can ignore the effect of the Berry phase term and replace $G_{\uparrow}$ by $G_{\uparrow}^{(0)}$. We then find

$$
\begin{aligned}
S_{1}[\boldsymbol{\Omega}] & =-\frac{1}{U} \sum_{\mathbf{r}, \mathbf{r}^{\prime}} \int d \tau \hat{t}_{\mathbf{r} \uparrow, \mathbf{r}^{\prime} \downarrow} \hat{t}_{\mathbf{r}^{\prime} \downarrow, \mathbf{r} \uparrow} \\
& =\frac{1}{2 U} \sum_{\mathbf{r}, \mathbf{r}^{\prime}} t_{\mathbf{r} \mathbf{r}^{\prime}}^{2} \int d \tau\left(\boldsymbol{\Omega}_{\mathbf{r}} \cdot \boldsymbol{\Omega}_{\mathbf{r}^{\prime}}-1\right) .
\end{aligned}
$$

In Eq. (3.40), the exchange interaction is assumed to be instantaneous, since the characteristic spin fluctuation energy $J=4 t^{2} / U$ is much smaller than $U$. The last line of Eq. (3.40) is obtained from $\hat{t}_{\mathbf{r} \uparrow, \mathbf{r}^{\prime} \downarrow} \hat{t}_{\mathbf{r}^{\prime} \downarrow, \mathbf{r} \uparrow}=$ $\left(1-\boldsymbol{\Omega}_{\mathbf{r}} \cdot \boldsymbol{\Omega}_{\mathbf{r}^{\prime}}\right) / 2.39$ Since $S_{1}^{\prime}$ vanishes at half-filling, while $S_{1}^{\prime \prime}$ turns out to be of order $O\left(t^{3} / U^{3}\right)$, we verify that the effective action of the spin degrees of freedom at order $O(t / U), S_{B}+S_{1}$, is nothing but the action of the (quantum) AF Heisenberg model expressed in terms of spin coherent states:

$$
S_{\mathrm{Heis}}[\boldsymbol{\Omega}]=S_{B}[\boldsymbol{\Omega}]+J \sum_{\left\langle\mathbf{r}, \mathbf{r}^{\prime}\right\rangle} \int d \tau\left(\frac{\boldsymbol{\Omega}_{\mathbf{r}} \cdot \boldsymbol{\Omega}_{\mathbf{r}^{\prime}}}{4}-\frac{1}{4}\right) .
$$

(a)

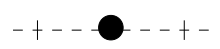

(b)

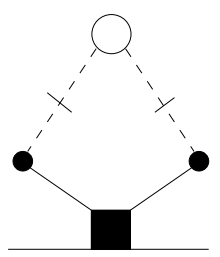

(c)

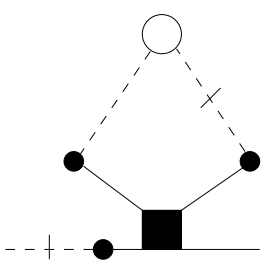

FIG. 10. Diagrammatic representation of the LHB self-energy at order $O(t / U)$. (a) $\hat{\Sigma}_{1}$. (b) $\hat{\Sigma}_{1}^{\prime}$. (c) $\hat{\Sigma}_{1}^{\prime \prime}$ (the symmetric diagram is not shown). Slashed dashed lines indicate interband transitions.

The self-energy $\hat{\Sigma}$ of particles in the LHB is defined by $\hat{\mathcal{G}}_{\uparrow}^{-1}=\hat{\mathcal{G}}_{\uparrow}^{(0)-1}-\hat{\Sigma}$, where $\hat{\mathcal{G}_{\uparrow}}$ is the propagator for a given configuration of $\boldsymbol{\Omega}$. It has three contributions to first order in $t / U$ (Fig. 10):

$$
\begin{aligned}
\hat{\Sigma}_{1}\left(a, a^{\prime}\right)= & \sum_{b, b^{\prime}} \hat{t}_{\uparrow \downarrow}(a, b) G_{\downarrow}\left(b, b^{\prime}\right) \hat{t}_{\downarrow \uparrow}\left(b^{\prime}, a^{\prime}\right), \\
\hat{\Sigma}_{1}^{\prime}\left(a, a^{\prime}\right)= & \sum_{b, b^{\prime}, b_{i}} \Gamma_{\uparrow \downarrow, \uparrow \downarrow}^{\mathrm{II}}\left(a, b ; a^{\prime}, b^{\prime}\right) G_{\downarrow}\left(b^{\prime}, b_{1}\right) \hat{t}_{\downarrow \uparrow}\left(b_{1}, b_{2}\right) \hat{\mathcal{G}}_{\uparrow}^{(0)}\left(b_{2}, b_{3}\right) \hat{t}_{\uparrow \downarrow}\left(b_{3}, b_{4}\right) G_{\downarrow}\left(b_{4}, b\right), \\
\hat{\Sigma}_{1}^{\prime \prime}\left(a, a^{\prime}\right)= & \sum_{b, b^{\prime}} \sum_{a_{i}, b_{i}} \hat{t}_{\uparrow \downarrow}\left(a, a_{1}\right) G_{\downarrow}\left(a_{1}, a_{2}\right) \Gamma_{\uparrow \downarrow, \uparrow \downarrow}^{\mathrm{II}}\left(b, a_{2} ; a^{\prime}, b^{\prime}\right) G_{\downarrow}\left(b^{\prime}, b_{1}\right) \hat{t}_{\downarrow \uparrow}\left(b_{1}, b_{2}\right) \hat{\mathcal{G}}_{\uparrow}^{(0)}\left(b_{2}, b_{3}\right) \hat{t}_{\uparrow \uparrow}\left(b_{3}, b_{4}\right) G_{\uparrow}\left(b_{4}, b\right) \\
& +\sum_{b, b^{\prime}} \sum_{a_{i}, b_{i}} \Gamma_{\uparrow \downarrow, \uparrow \downarrow}^{\mathrm{II}}\left(a, b ; b^{\prime}, a_{2}\right) G_{\uparrow}\left(b^{\prime}, b_{1}\right) \hat{t}_{\uparrow \uparrow}\left(b_{1}, b_{2}\right) \hat{\mathcal{G}}_{\uparrow}^{(0)}\left(b_{2}, b_{3}\right) \hat{t}_{\uparrow \downarrow}\left(b_{3}, b_{4}\right) G_{\downarrow}\left(b_{4}, b\right) G_{\downarrow}\left(a_{2}, a_{1}\right) \hat{t}_{\downarrow \uparrow}\left(a_{1}, a^{\prime}\right) .
\end{aligned}
$$

$\hat{\Sigma}_{1}$ describes indirect hopping processes between nextnearest-neighbor sites which occur via virtual transitions to the UHB. In the $t-J$ model, these processes show up in the pair-hopping term.

So far our discussion has been confined to a formal framework. For practical calculations, further approximations will be necessary. Whereas the fermionic fields $\gamma_{\sigma}$ can be integrated out in a systematic $t / U$ expansion, one still has to carry out the functional integral over the unit vector field $\boldsymbol{\Omega}$.

The simplest approach consists in expanding around a broken-symmetry ground-state by making a saddle point approximation on the spin variables $\boldsymbol{\Omega}_{\mathbf{r}}$. Different choices, corresponding to AF, ferromagnetic or spiral orders, are possible. This approach, which is discussed at length in Ref. 11, can be justified by taking a "large- $S$ " semiclassical limit of the Hubbard model. The spin- $\frac{1}{2}$ coherent states can be promoted to spin- $S$ coherent states 
(with $S$ arbitrary) by writing the $\mathrm{SU}(2) / \mathrm{U}(1)$ rotation matrix $R_{\mathbf{r}}$ as in Eq. (3.36) and generalizing the constraint $\left|z_{\mathbf{r} \uparrow}\right|^{2}+\left|z_{\mathbf{r} \downarrow}\right|^{2}=1$ to $\left|z_{\mathbf{r} \uparrow}\right|^{2}+\left|z_{\mathbf{r} \downarrow}\right|^{2}=2 S$. The latter equality allows to write $z_{\mathbf{r} \uparrow}=\sqrt{2 S} \cos \left(\theta_{\mathbf{r}} / 2\right) e^{-\frac{i}{2}\left(\varphi_{\mathbf{r}}+\psi_{\mathbf{r}}\right)}$ and $z_{\mathbf{r} \downarrow}=\sqrt{2 S} \sin \left(\theta_{\mathbf{r}} / 2\right) e^{\frac{i}{2}\left(\varphi_{\mathbf{r}}-\psi_{\mathbf{r}}\right)}$ where the choice of $\psi_{\mathbf{r}}$ is free and corresponds to the U(1) gauge freedom. The action of the Hubbard model is obtained from (3.29) with the replacement $A^{0} \rightarrow 2 S A^{0}$ and $\hat{t} \rightarrow 2 S \hat{t}$. [Note that the creation of a (fermionic) hole corresponds to a total removal of the local moment.] In the limit $S \rightarrow \infty$ (with $t S=$ constant), the Berry phase term suppresses quantum fluctuations of $\boldsymbol{\Omega}$. The spin variables become classical and do not fluctuate at zero temperature. The leading correction in $1 / S$ gives the spin-wave modes around the broken-symmetry ground-state.

At half-filling, one can also try to compute $\mathcal{G}$ by directly averaging $\hat{\mathcal{G}}$ with $S[\boldsymbol{\Omega}]$ [see Eq. (3.32)], since the effective action of the spin degrees of freedom, $S_{\mathrm{Heis}}[\boldsymbol{\Omega}]$, can be analyzed by various methods. 43 Since $S_{\text {Heis }}[\Omega]$ is not a Gaussian action, this seems a priori a very difficult task. We show in Ref. 42 how this technical difficulty can be circumvented by using the Schwinger-boson meanfield theory of the Heisenberg model. The advantage of this approach is that one starts from a good description of the magnetic properties of the system (which are described by $S_{\text {Heis }}$ ): absence of long-range order at finite temperature, exponential divergence in $1 / T$ of the magnetic correlation length, etc. Moreover, this formulation is a good starting point to study how spin fluctuations affect hole motion. 42

It should be pointed out that Eqs. (3.32) and (3.33) cast the Hubbard model in the form of a spin-fermion model, which can be seen as the strong-coupling counterpart of the "weak-comling" spin-fermion model that has been studied so far.10 There are however important differences between the latter and our formalism: (i) In the "weak-coupling" spin-fermion model, the effective action of the spin degrees of freedom is generally assumed to be Gaussian. In our approach, the effective action $S[\Omega]$ is obtained exactly (to order $O(t / U)$ ) by integrating out the fermionic degrees of freedom. (ii) The spinfermion model omits coupling between spin fluctuations and charge degrees of freedom. Fermions interact with spin fluctuations via a spin-spin interaction. In our approach, there is an intimate coupling between charge degrees of freedom (i.e. hole motion) and spin fluctuations, which shows up in the $\boldsymbol{\Omega}$-dependence of the intersite hopping matrix. (iii) The spin-fermion model is restricted to the weak-coupling regime, whereas our approach is appropriate to the strong-coupling regime.56

Finally, we would like to discuss the relation to the $t$ $J$ model. Starting from the effective action (3.29) and integrating out the $\mathrm{UHB}$, one recovers the action of the $t$ - $J$ model in the spin-hole coherent-state path integral.52 A further change of variables yields the action of the $t-J$ model in the slave fermion formalism.57 [See Sec. III.B.3 of Ref. 39.] The connection between the action (3.29) and the $t$ - $J$ model is however not straightforward and involves some subtleties regarding the proper time ordering in the functional integral. $\$ 9$ As a result, both formulations should be considered as two different descriptions of the strong-coupling limit of the Hubbard model. For instance, indirect hopping processes between nextnearest neighbors appear in the Gaussian part of the action (3.29), while they are described by the pair-hopping term in the $t-J$ model (this term involves a three-body interaction in the standard formulation of the $t-J$ model). But the most interesting aspect of our formulation is the use of spin-particle-hole coherent states. By clearly distinguishing between charge and spin degrees of freedom, spin-particle-hole coherent states open up new possibilities for analyzing hole motion in presence of strong spin fluctuations. 42

\section{SUMMARY AND CONCLUSION}

There are two main ingredients in the derivation of the Heisenberg model from the Hubbard model at half-filling: First, one should deal with spin operators (instead of the original fermionic operators) in order to account for the local moments that appear in the Mott insulating phase. Then, the effective Hamiltonian for these spinoperators is derived by expanding with respect to $t / U$.27

In this paper, we have proposed a generalization of this procedure to the doped case. Our approach is based on the introduction of spin-particle-hole coherent states which generalize the spin- $\frac{1}{2}$ coherent states by allowing the creation of a hole or an additional particle. They also generalize the spin-hole cpherent states introduced in the context of the $t-J$ model. 34 The spin-particle-hole coherent states can be used to derive a path integral and naturally lead to a spin-rotation-invariant slave-boson formulation of the Hubbard model (Sec. II). By performing two successive Hubbard-Stratonovich transformations of the intersite hopping term, the path integral can be recast in a from which is suitable for a perturbation expansion in $t / U$. The action $S\left[\gamma^{*}, \gamma ; \boldsymbol{\Omega}\right]$ can be expressed in terms of two Grassmann variables $\left(\gamma_{\uparrow}, \gamma_{\downarrow}\right)$ and a unit vector field. A singly occupied site is described by a spin- $\frac{1}{2}$ coherent state $\left|\boldsymbol{\Omega}_{\mathbf{r}}\right\rangle$, while $\gamma_{\uparrow}\left(\gamma_{\downarrow}\right)$ describes a particle propagating in the LHB (UHB). The fermionic action has interaction terms to all orders, given by the atomic vertices $\Gamma^{R}$ [Eq. (3.24)]. The fermions also interact with spin fluctuations which show up in the dynamics of $\boldsymbol{\Omega}$. In the strong-coupling limit $t \ll U$, the action can be truncated to quartic order in the fermionic field [Eq. (3.29)] and used as the starting point of a diagrammatic expansion. At half-filling and to order $O(t / U)$, we recover the action of the (quantum) Heisenberg model.

Since the fermionic field can be systematically integrated out within a $t / U$ expansion, the main practical difficulty comes from the dynamics of $\boldsymbol{\Omega}$ (spin fluctuations). The simplest approach consists in expanding 
about a broken-symmetry ground-state my making a saddle point approximation on the spin variables $\boldsymbol{\Omega}_{\mathbf{r}}$. Different choices, corresponding to AF, ferromagnetic, or spiral orders are possible. This approach is discussed in detail in Ref. 41. The spin-particle-hole coherent-state path integral turns out to be a very convenient tool for studying spin waves about a broken-symmetry ground-state in the strong-coupling limit.

The main characteristic of the spin-particle-hole coherent-state path integral lies in the clear distinction between charge $(\gamma)$ and spin $(\boldsymbol{\Omega})$ degrees of freedom. This opens up new possibilities for the computation of the Green's functions. One can first calculate the Green's functions for a given (time-dependent) configuration of $\boldsymbol{\Omega}$ and then perform the average with the effective action $S[\boldsymbol{\Omega}]$ of the spin degrees of freedom [see Sec. III D and Eqs. (3.32 3.33)]. This program is carried out in Ref. 42.

\section{APPENDIX A: PATH INTEGRAL FOR THE SINGLE-SITE HUBBARD MODEL}

Introducing $(M-1)$ times the resolution of the identity (2.13) in Eq. 2.22), one obtains

$$
Z_{\text {at }}=\mathcal{N}^{M} \int\left(\prod_{k=1}^{M} \frac{d \boldsymbol{\Omega}_{k}}{4 \pi} d \zeta_{k}^{*} d \zeta_{k}\right) e^{-\sum_{k=1}^{M} \alpha\left|\zeta_{k}\right|^{2}} \prod_{k=1}^{M}\left\langle\boldsymbol{\Omega}_{k}, \zeta_{k}\left|\hat{P}_{k} e^{-\epsilon(\hat{H}-\mu \hat{N})}\right| \boldsymbol{\Omega}_{k-1}, \zeta_{k-1}\right\rangle,
$$

with $\epsilon=\beta / M$ and the boundary conditions: $\boldsymbol{\Omega}_{0}=\boldsymbol{\Omega}_{M}, e_{M}=e_{0}, p_{M}=p_{0}, d_{M}=d_{0}$, and $f_{\sigma M}=-f_{\sigma 0}$. The projection operator $\hat{P}_{k}$ is defined by Eq. 2.15) with the spin-quantization axis determined by the unit vector $\boldsymbol{\Omega}_{k}$. It can be written as

$$
\hat{P}_{k}=\int_{0}^{\frac{2 \pi}{\epsilon}} \frac{\epsilon d \lambda_{k}^{(1)}}{2 \pi} e^{-i \epsilon \lambda_{k}^{(1)} \hat{Q}_{k}^{(1)}} \prod_{\sigma} \int_{0}^{\frac{2 \pi}{\epsilon}} \frac{\epsilon d \lambda_{\sigma k}^{(2)}}{2 \pi} e^{-i \epsilon \lambda_{\sigma k}^{(2)} \hat{Q}_{\sigma k}^{(2)}}
$$

Since the constraints $\hat{Q}^{(1)}$ and $\hat{Q}_{\sigma}^{(2)}$ commute with the Hamiltonian $\hat{H}-\mu \hat{N}$, we obtain

$$
\begin{aligned}
Z_{\mathrm{at}} & =\mathcal{N}^{M} \int\left(\prod_{k=1}^{M} \frac{d \boldsymbol{\Omega}_{k}}{4 \pi} d \zeta_{k}^{*} d \zeta_{k} d \lambda_{k}\right) e^{-\sum_{k=1}^{M} \alpha\left|\zeta_{k}\right|^{2}} \prod_{k=1}^{M}\left\langle\boldsymbol{\Omega}_{k}, \zeta_{k}\left|\hat{P}_{k} e^{-\epsilon \hat{K}_{k}}\right| \boldsymbol{\Omega}_{k-1}, \zeta_{k-1}\right\rangle \\
& \simeq \mathcal{N}^{M} \int\left(\prod_{k=1}^{M} \frac{d \boldsymbol{\Omega}_{k}}{4 \pi} d \zeta_{k}^{*} d \zeta_{k} d \lambda_{k}\right) e^{-\sum_{k=1}^{M} \alpha\left|\zeta_{k}\right|^{2}} \prod_{k=1}^{M}\left\langle\boldsymbol{\Omega}_{k}, \zeta_{k} \mid \boldsymbol{\Omega}_{k-1}, \zeta_{k-1}\right\rangle e^{-\epsilon K_{k, k-1}}
\end{aligned}
$$

in the limit $\epsilon \rightarrow 0$, where

$$
\begin{aligned}
\int d \lambda_{k} & \equiv \int_{0}^{\frac{2 \pi}{\epsilon}} \frac{\epsilon d \lambda_{k}^{(1)}}{2 \pi} \prod_{\sigma} \int_{0}^{\frac{2 \pi}{\epsilon}} \frac{\epsilon d \lambda_{\sigma k}^{(2)}}{2 \pi} \\
\hat{K}_{k} & =\hat{H}-\mu \hat{N}+i \lambda^{(1)} \hat{Q}_{k}^{(1)}+i \sum_{\sigma} \lambda_{\sigma k}^{(2)} \hat{Q}_{\sigma}^{(2)} \\
K_{k, k-1} & =\frac{\left\langle\boldsymbol{\Omega}_{k}, \zeta_{k}\left|\hat{K}_{k}\right| \boldsymbol{\Omega}_{k-1}, \zeta_{k-1}\right\rangle}{\left\langle\boldsymbol{\Omega}_{k}, \zeta_{k} \mid \boldsymbol{\Omega}_{k-1}, \zeta_{k-1}\right\rangle}
\end{aligned}
$$

Using the expression of the scalar product [Eq. (2.12)], the partition function is finally written as

$$
Z_{\text {at }}=\mathcal{N}^{M} \int\left(\prod_{k=1}^{M} \frac{d \boldsymbol{\Omega}_{k}}{4 \pi} d \zeta_{k}^{*} d \zeta_{k} d \lambda_{k}\right) \exp \left\{\sum_{k=1}^{M}\left[-\alpha\left|\zeta_{k}\right|^{2}+\zeta_{k}^{*} \zeta_{k-1}+f_{\uparrow k}^{*} p_{k}^{*} f_{\uparrow k-1} p_{k-1}\left(\left\langle\boldsymbol{\Omega}_{k} \mid \boldsymbol{\Omega}_{k-1}\right\rangle-1\right)-\epsilon K_{k, k-1}\right]\right\} .
$$

Taking $\alpha_{e}=\alpha_{d}=1$ and neglecting the overall normalization constant $\mathcal{N}^{M}$ (see the discussion in section IIB), there is now no difficulty to take the continuum time limit. $K_{k, k-1} \simeq K_{k, k}$ is evaluated by writing the Hamiltonian as

$$
\hat{H}-\mu \hat{N}=U \hat{d}^{\dagger} \hat{d}-\mu \sum_{\sigma} \hat{f}_{\sigma}^{\dagger} \hat{f}_{\sigma}
$$


where the spin-quantization axis $\boldsymbol{\Omega}_{k}$ is chosen. The constraints $\hat{Q}^{(1)}$ and $\hat{Q}_{\sigma}^{(2)}$ being also defined with respect to the spin-quantization axis $\boldsymbol{\Omega}_{k}$, we obtain

$$
\begin{aligned}
K_{k, k}= & U\left|d_{k}\right|^{2}-\mu \sum_{\sigma} f_{\sigma k}^{*} f_{\sigma k}+i \lambda_{k}^{(1)}\left(\left|e_{k}\right|^{2}+\left|p_{k}\right|^{2}+\left|d_{k}\right|^{2}-1\right) \\
& +i \lambda_{\uparrow k}^{(2)}\left(f_{\uparrow k}^{*} f_{\uparrow k}-\left|p_{k}\right|^{2}-\left|d_{k}\right|^{2}\right)+i \lambda_{\downarrow k}^{(2)}\left(f_{\downarrow k}^{*} f_{\downarrow k}-\left|d_{k}\right|^{2}\right) .
\end{aligned}
$$

We also note that $\left\langle\boldsymbol{\Omega}_{k} \mid \boldsymbol{\Omega}_{k-1}\right\rangle-1=\left\langle\boldsymbol{\Omega}_{k} \mid \boldsymbol{\Omega}_{k-1}\right\rangle-\left\langle\boldsymbol{\Omega}_{k} \mid \boldsymbol{\Omega}_{k}\right\rangle$ yields a term $-\langle\boldsymbol{\Omega} \mid \dot{\boldsymbol{\Omega}}\rangle$ in the continuum time limit. Here the dot denotes a time derivative. The final expression of the action $S_{\text {at }}$ in the atomic limit is given by Eqs. (2.29).

\section{APPENDIX B: EVALUATION OF $\left\langle\boldsymbol{\Omega}, \zeta\left|\hat{C}_{\mathbf{R}}^{\dagger} \hat{C}_{\mathbf{R}^{\prime}}\right| \boldsymbol{\Omega}, \zeta\right\rangle$}

In this appendix, we prove Eq. 2.31) (we do not write explicitly the time index $k$ ). We introduce the operator

$$
\hat{\phi}_{\mathbf{r} \sigma}^{\dagger}=\sum_{\sigma^{\prime}}\left(R_{\mathbf{r}}\right)_{\sigma^{\prime} \sigma} \hat{c}_{\mathbf{r} \sigma^{\prime}}^{\dagger}
$$

which creates a particle with spin $\sigma$ in the spin reference frame determined by $\boldsymbol{\Omega}_{\mathbf{r}}$. In the space spanned by $\mathcal{B}_{\left\{\boldsymbol{\Omega}_{\mathbf{r}}\right\}}^{\prime}$, we have $\hat{\phi}_{\mathbf{r} \sigma}=\hat{\gamma}_{\mathbf{r} \sigma}$. We then deduce

$$
\begin{aligned}
\left\langle\boldsymbol{\Omega}, \zeta\left|\hat{c}_{\mathbf{r}}^{\dagger} \hat{\mathbf{c}}_{\mathbf{r}^{\prime}}\right| \boldsymbol{\Omega}, \zeta\right\rangle & =\sum_{\sigma, \sigma^{\prime}}\left\langle\boldsymbol{\Omega}, \zeta\left|\hat{\gamma}_{\mathbf{r} \sigma}^{\dagger}\left(R_{\mathbf{r}}^{\dagger} R_{\mathbf{r}^{\prime}}\right)_{\sigma \sigma^{\prime}} \hat{\gamma}_{\mathbf{r}^{\prime} \sigma^{\prime}}\right| \boldsymbol{\Omega}, \zeta\right\rangle \\
& =\gamma_{\mathbf{r}}^{\dagger} R_{\mathbf{r}}^{\dagger} R_{\mathbf{r}^{\prime}} \gamma_{\mathbf{r}^{\prime}}\langle\boldsymbol{\Omega}, \zeta \mid \boldsymbol{\Omega}, \zeta\rangle,
\end{aligned}
$$

where $\gamma_{\mathbf{r} \uparrow}=e_{\mathbf{r}}^{*} p_{\mathbf{r}} f_{\mathbf{r} \uparrow}$ and $\gamma_{\mathbf{r} \downarrow}=p_{\mathbf{r}}^{*} d_{\mathbf{r}} f_{\mathbf{r} \downarrow}$. The intersite term in the action (2.34) follows from Eq. (B2).

\section{APPENDIX C: GREEN'S FUNCTIONS IN THE ATOMIC LIMIT}

In this section we compute the connected atomic Green's functions

$$
G_{\left\{a_{i}, b_{i}\right\}}^{R c}=(-1)^{R}\left\langle T_{\tau} \hat{\gamma}_{a_{1}} \cdots \hat{\gamma}_{a_{R}} \hat{\gamma}_{b_{R}}^{\dagger} \cdots \hat{\gamma}_{b_{1}}^{\dagger}\right\rangle_{\text {at }, \mathrm{c}}
$$

written here in the operator formalism ( $T_{\tau}$ is the imaginary-time ordering operator). We consider a single site and drop the site index.

Let us first consider the Green's functions determined by $S_{\mathrm{at}}^{(0)}$. In the basis $\mathcal{B}_{\Omega}^{\prime}$, the operators $\hat{\gamma}_{\sigma}$ and the Hamiltonian $\hat{H}-\mu \hat{N}$ can be written as (in matrix form)

$$
\begin{aligned}
\hat{\gamma}_{\uparrow} & =\left(\begin{array}{lll}
0 & 1 & 0 \\
0 & 0 & 0 \\
0 & 0 & 0
\end{array}\right), \hat{\gamma}_{\downarrow}=\left(\begin{array}{ccc}
0 & 0 & 0 \\
0 & 0 & -1 \\
0 & 0 & 0
\end{array}\right), \\
\hat{H}-\mu \hat{N} & =\left(\begin{array}{ccc}
0 & 0 & 0 \\
0 & -\mu & 0 \\
0 & 0 & U-2 \mu
\end{array}\right) .
\end{aligned}
$$

We easily find the time dependence of the operators $\hat{\gamma}_{\sigma}^{(\dagger)}(\tau)=\hat{U}(-\tau) \hat{\gamma}_{\sigma}^{(\dagger)} \hat{U}(\tau)$ (where $\hat{U}(\tau)=e^{-\tau(\hat{H}-\mu \hat{N})}$ is the evolution operator):

$$
\begin{aligned}
& \hat{\gamma}_{\uparrow}(\tau)=e^{\mu \tau} \hat{\gamma}_{\uparrow}, \quad \hat{\gamma}_{\uparrow}^{\dagger}(\tau)=e^{-\mu \tau} \hat{\gamma}_{\uparrow}^{\dagger}, \\
& \hat{\gamma}_{\downarrow}(\tau)=e^{(\mu-U) \tau} \hat{\gamma}_{\downarrow}, \quad \hat{\gamma}_{\downarrow}^{\dagger}(\tau)=e^{-(\mu-U) \tau} \hat{\gamma}_{\downarrow}^{\dagger} .
\end{aligned}
$$

The single-particle Green's function $G_{\sigma}^{(0)}(\tau)=-\left\langle T_{\tau} \hat{\gamma}(\tau) \hat{\gamma}_{\sigma}^{\dagger}(\tau)\right\rangle$ is found to be 


$$
\begin{aligned}
& G_{\uparrow}^{(0)}(\tau)=\frac{e^{\mu \tau}}{Z_{\mathrm{at}}^{(0)}}\left[\theta(-\tau) e^{\beta \mu}-\theta(\tau)\right] \simeq e^{\mu \tau} \theta(-\tau), \\
& G_{\downarrow}^{(0)}(\tau)=\frac{e^{(\mu-U) \tau}}{Z_{\mathrm{at}}^{(0)}}\left[\theta(-\tau) e^{\beta(2 \mu-U)}-\theta(\tau) e^{\beta \mu}\right] \simeq-e^{(\mu-U) \tau} \theta(\tau) .
\end{aligned}
$$

The final expressions are obtained in the low-temperature limit and we have used $Z_{\text {at }}^{(0)}=1+e^{\beta \mu}+e^{\beta(2 \mu-U)} \simeq e^{\beta \mu}$. The latter approximation neglects the contribution of the empty and doubly occupied sites, in agreement with the discussion of Sec. IIB. As expected, $Z_{\mathrm{at}}^{(0)}$ is the partition function for a given spin direction. In Fourier space, we obtain

$$
\begin{aligned}
& G_{\uparrow}^{(0)}(i \omega)=(i \omega+\mu)^{-1}, \\
& G_{\downarrow}^{(0)}(i \omega)=(i \omega+\mu-U)^{-1} .
\end{aligned}
$$

In the same way, we can calculate the two-particle Green's function. For instance, we have

$$
\begin{aligned}
G_{\uparrow \uparrow, \uparrow \uparrow}^{\mathrm{II}}\left(\tau_{1}, \tau_{2} ; \tau_{3}, \tau_{4}\right)= & \frac{1}{Z_{\mathrm{at}}^{(0)}} e^{\mu\left(\tau_{1}+\tau_{2}-\tau_{3}-\tau_{4}\right)} \operatorname{Tr}\left[\hat{U}(\beta)\left(\hat{\gamma}_{\uparrow}^{\dagger} \hat{\gamma}_{\uparrow}\right)^{2}\right] \\
& \times\left[\theta\left(\tau_{4}-\tau_{2}\right) \theta\left(\tau_{2}-\tau_{3}\right) \theta\left(\tau_{3}-\tau_{1}\right)-\theta\left(\tau_{3}-\tau_{2}\right) \theta\left(\tau_{2}-\tau_{4}\right) \theta\left(\tau_{4}-\tau_{1}\right)\right. \\
& \left.-\theta\left(\tau_{4}-\tau_{1}\right) \theta\left(\tau_{1}-\tau_{3}\right) \theta\left(\tau_{3}-\tau_{2}\right)+\theta\left(\tau_{3}-\tau_{1}\right) \theta\left(\tau_{1}-\tau_{4}\right) \theta\left(\tau_{4}-\tau_{2}\right)\right]
\end{aligned}
$$

where $\operatorname{Tr}\left[\hat{U}(\beta)\left(\hat{\gamma}_{\uparrow}^{\dagger} \hat{\gamma}_{\uparrow}\right)^{2}\right]=e^{\beta \mu}$. We do not distinguish between $G^{\mathrm{II}(0)}$ and $G^{\mathrm{II}}$, since the effect of the Berry phase term on $G^{R c}(\geq 2)$ can be ignored (see Sec. III]). One can verify that $G_{\uparrow \uparrow, \uparrow \uparrow}^{\mathrm{II}}\left(\tau_{1}, \tau_{2} ; \tau_{3}, \tau_{4}\right)$ coincides with its disconnected part

$$
\begin{aligned}
G_{\uparrow \uparrow, \uparrow \uparrow}^{\mathrm{IIdis}}\left(\tau_{1}, \tau_{2} ; \tau_{3}, \tau_{4}\right) & =G_{\uparrow}^{(0)}\left(\tau_{1}-\tau_{3}\right) G_{\uparrow}^{(0)}\left(\tau_{2}-\tau_{4}\right)-G_{\uparrow}^{(0)}\left(\tau_{1}-\tau_{4}\right) G_{\uparrow}^{(0)}\left(\tau_{2}-\tau_{3}\right) \\
& =e^{\mu\left(\tau_{1}+\tau_{2}-\tau_{3}-\tau_{4}\right)}\left[\theta\left(-\tau_{1}+\tau_{3}\right) \theta\left(-\tau_{2}+\tau_{4}\right)-\theta\left(-\tau_{1}+\tau_{4}\right) \theta\left(-\tau_{2}+\tau_{3}\right)\right]
\end{aligned}
$$

so that $G_{\uparrow \uparrow, \uparrow \uparrow}^{\mathrm{II} c}=0$. Similarly $G_{\downarrow \downarrow, \downarrow \downarrow}^{\mathrm{II} c}=0$. By using the definition of the third cumulant

$$
\begin{aligned}
G_{a_{1} a_{2} a_{3}, b_{1} b_{2} b_{3}}^{\mathrm{III} c} & \left.\frac{\delta^{(6)} W\left[\psi^{*}, \psi ; \Omega\right]}{\delta \psi_{a_{1}}^{*} \cdots \delta \psi_{b_{1}}}\right|_{\psi^{*}=\psi=0} \\
= & G_{a_{1} a_{2} a_{3}, b_{1} b_{2} b_{3}}^{\mathrm{III}}-G_{a_{1} b_{1}}^{(0)} G_{a_{2} a_{3}, b_{2} b_{3}}^{\mathrm{II} c}-G_{a_{2} b_{2}}^{(0)} G_{a_{1} a_{3}, b_{1} b_{3}}^{\mathrm{II} c}-G_{a_{3} b_{3}}^{(0)} G_{a_{1} a_{2}, b_{1} b_{2}}^{\mathrm{II} c}+G_{a_{2} b_{1}}^{(0)} G_{a_{1} a_{3}, b_{2} b_{3}}^{\mathrm{II} c} \\
& -G_{a_{3} b_{1}}^{(0)} G_{a_{1} a_{2}, b_{2} b_{3}}^{\mathrm{II} c}+G_{a_{3} b_{2}}^{(0)} G_{a_{1} a_{2}, b_{1} b_{3}}^{\mathrm{II} c}+G_{a_{1} b_{2}}^{(0)} G_{a_{2} a_{3}, b_{1} b_{3}}^{\mathrm{II} c}+G_{a_{2} b_{3}}^{(0)} G_{a_{1} a_{3}, b_{1} b_{2}}^{\mathrm{II} c}-G_{a_{1} b_{3}}^{(0)} G_{a_{2} a_{3}, b_{1} b_{2}}^{\mathrm{II} c} \\
& -G_{a_{1} b_{1}}^{(0)} G_{a_{2} b_{2}}^{(0)} G_{a_{3} b_{3}}^{(0)}+G_{a_{1} b_{2}}^{(0)} G_{a_{2} b_{1}}^{(0)} G_{a_{3} b_{3}}^{(0)}+G_{a_{1} b_{1}}^{(0)} G_{a_{2} b_{3}}^{(0)} G_{a_{3} b_{2}}^{(0)} \\
& -G_{a_{1} b_{2}}^{(0)} G_{a_{2} b_{3}}^{(0)} G_{a_{3} b_{1}}^{(0)}-G_{a_{1} b_{3}}^{(0)} G_{a_{2} b_{1}}^{(0)} G_{a_{3} b_{2}}^{(0)}+G_{a_{1} b_{3}}^{(0)} G_{a_{2} b_{2}}^{(0)} G_{a_{3} b_{1}}^{(0)},
\end{aligned}
$$

one also obtains $G_{\sigma \sigma \sigma, \sigma \sigma \sigma}^{\mathrm{III} c}=0$. [Note that this verification requires to consider $6 !=360$ different time sectors.] It is clear that this result holds to all orders, i.e.

$$
G_{\sigma \cdots \sigma, \sigma \cdots \sigma}^{R c}=0
$$

The Green's function $G_{\uparrow \downarrow, \uparrow \downarrow}^{\mathrm{II} c}$ can be calculated in the same way:

$$
\begin{aligned}
G_{\uparrow \downarrow, \uparrow \downarrow}^{\mathrm{II}}\left(\tau_{1}, \tau_{2} ; \tau_{3}, \tau_{4}\right)= & e^{\mu\left(\tau_{1}-\tau_{3}\right)+(\mu-U)\left(\tau_{2}-\tau_{4}\right)}\left\{e^{-\beta \mu} \theta\left(\tau_{1}-\tau_{2}\right) \theta\left(\tau_{2}-\tau_{4}\right) \theta\left(\tau_{4}-\tau_{3}\right)\right. \\
& \left.-\theta\left(\tau_{2}-\tau_{4}\right) \theta\left(\tau_{3}-\tau_{1}\right)\left[\theta\left(\tau_{1}-\tau_{2}\right)+\theta\left(\tau_{4}-\tau_{3}\right)\right]+e^{\beta(\mu-U)} \theta\left(\tau_{4}-\tau_{3}\right) \theta\left(\tau_{3}-\tau_{1}\right) \theta\left(\tau_{1}-\tau_{2}\right)\right\} .
\end{aligned}
$$

In Fourier space, we obtain from Eq. (C10) the connected part

$$
\begin{aligned}
G_{\uparrow \downarrow, \uparrow \downarrow}^{\mathrm{II} c}\left(i \omega_{1}, i \omega_{2} ; i \omega_{3}\left(i \omega_{4}\right)\right)= & -G_{\uparrow}^{(0)}\left(i \omega_{1}\right) G_{\downarrow}^{(0)}\left(i \omega_{2}\right) G_{\uparrow}^{(0)}\left(i \omega_{3}\right) G_{\downarrow}^{(0)}\left(i \omega_{4}\right)\left(i \omega_{2}-i \omega_{3}-U\right) \\
& -\frac{1}{i \omega_{1}+i \omega_{2}+2 \mu-U}\left[e^{-\beta \mu} G_{\uparrow}^{(0)}\left(i \omega_{1}\right) G_{\uparrow}^{(0)}\left(i \omega_{3}\right)-e^{\beta(\mu-U)} G_{\downarrow}^{(0)}\left(i \omega_{2}\right) G_{\downarrow}^{(0)}\left(i \omega_{4}\right)\right],
\end{aligned}
$$


where $\omega_{4}=\omega_{1}+\omega_{2}-\omega_{3}$ is fixed by energy conservation. $G^{\mathrm{IIc}}$ is related to the two-particle vertex by

$$
G_{\uparrow \downarrow, \uparrow \downarrow}^{\mathrm{IIc} c}\left(i \omega_{1}, i \omega_{2} ; i \omega_{3}\left(i \omega_{4}\right)\right)=-G_{\uparrow}^{(0)}\left(i \omega_{1}\right) G_{\downarrow}^{(0)}\left(i \omega_{2}\right) \Gamma_{\uparrow \downarrow, \uparrow \downarrow}\left(i \omega_{1}, i \omega_{2} ; i \omega_{3},\left(i \omega_{4}\right)\right) G_{\uparrow}^{(0)}\left(i \omega_{3}\right) G_{\downarrow}^{(0)}\left(i \omega_{4}\right) .
$$

From Eq. (C11), we then deduce

$$
\Gamma_{\uparrow \downarrow, \uparrow \downarrow}\left(i \omega_{1}, i \omega_{2} ; i \omega_{3}\left(i \omega_{4}\right)\right)=i \omega_{2}-i \omega_{3}-U
$$

in the low-temperature limit, i.e by neglecting the second and third terms of the rhs of Eq. (C11). These terms correspond to the contributions of the empty and doubly occupied sites, which, according to the discussion of Sec. II B, should be discarded.

Let us now consider the effect of the Berry phase term

$$
S_{\mathrm{at}}-S_{\mathrm{at}}^{(0)}=\int d \tau A^{0} f_{\uparrow}^{*} p^{*} f_{\uparrow} p
$$

on the single particle Green's function $G$. The latter can be calculated from

$$
\begin{aligned}
& G_{\uparrow}(\tau)=-\frac{1}{Z_{\mathrm{at}}^{(0)}} \int d \lambda \int \mathcal{D}[f, e, p, d] e^{*}(\tau) p(\tau) f_{\uparrow}(\tau) f_{\uparrow}^{*}(0) p^{*}(0) e(0) e^{-S_{\mathrm{at}}}, \\
& G_{\downarrow}(\tau)=-\frac{1}{Z_{\mathrm{at}}^{(0)}} \int d \lambda \int \mathcal{D}[f, e, p, d] d(\tau) p^{*}(\tau) f_{\downarrow}(\tau) f_{\downarrow}^{*}(0) p(0) d^{*}(0) e^{-S_{\mathrm{at}}}
\end{aligned}
$$

by integrating explicitly the bosonic $(e, p, d)$ and fermionic $\left(f_{\sigma}\right)$ fields and the Lagrange multipliers. The calculation can be found in Appendix $\mathrm{C}$ of Ref. 39. Neglecting the Berry phase term, one recovers the results obtained above [Eqs. (C4)]. The first order correction in $A^{0}=\langle\boldsymbol{\Omega} \mid \dot{\boldsymbol{\Omega}}\rangle$ is

$$
\begin{aligned}
& G_{\uparrow}^{(1)}(\tau)=\int d \tau_{1} G_{\uparrow}^{(0)}\left(\tau-\tau_{1}\right) A^{0}\left(\tau_{1}\right) G_{\uparrow}^{(0)}\left(\tau_{1}\right), \\
& G_{\downarrow}^{(1)}(\tau)=-\int d \tau_{1} G_{\downarrow}^{(0)}\left(\tau-\tau_{1}\right) A^{0}\left(\tau_{1}\right) G_{\downarrow}^{(0)}\left(\tau_{1}\right)
\end{aligned}
$$

in the limit $T \rightarrow 0$. These results are easily extrapolated to higher orders as (in matrix form)

$$
\begin{aligned}
& G_{\uparrow}=G_{\uparrow}^{(0)}+G_{\uparrow}^{(0)} A^{0} G_{\uparrow}^{(0)}+G_{\uparrow}^{(0)} A^{0} G_{\uparrow}^{(0)} A^{0} G_{\uparrow}^{(0)}+\cdots \\
& G_{\downarrow}=G_{\downarrow}^{(0)}-G_{\downarrow}^{(0)} A^{0} G_{\downarrow}^{(0)}+G_{\downarrow}^{(0)} A^{0} G_{\downarrow}^{(0)} A^{0} G_{\downarrow}^{(0)}-\cdots
\end{aligned}
$$

or, equivalently,

$$
\begin{aligned}
& G_{\uparrow}^{-1}=G_{\uparrow}^{(0)-1}-A^{0}, \\
& G_{\downarrow}^{-1}=G_{\downarrow}^{(0)-1}+A^{0} .
\end{aligned}
$$

Note that these Green's functions, like the gauge field $A^{0}$, depend on the site which is considered.

\section{APPENDIX D: CLOSED LOOPS IN THE STRONG-COUPLING PERTURBATIVE EXPANSION}

In this Appendix, we discuss the vanishing of closed loops of $\gamma_{\uparrow}$ particles in the strong-coupling perturbative expansion.

Since the Green's functions $G^{R c}$ are related to the vertices $\Gamma^{R}$, a diagram can always be expressed in terms of the $G^{R c}$ 's. Using Eqs. (C3), one can show that $G^{R c}$ is a sum of terms proportional to $(-1)^{P} \prod_{i=1}^{R} G_{\sigma_{i}}\left(\tau_{i}-\tau_{P(i)}\right)$ where $P$ is a permutation of $[1, R]$ satisfying $\sigma_{P(i)}=\sigma_{i}(i \in[1, R])$. Each closed loop of $\gamma_{\sigma}$ particles will then give terms of the type $G_{\sigma}\left(\tau_{0}-\tau_{1}\right) \hat{t}_{\sigma \sigma} \cdots \hat{t}_{\sigma \sigma} G_{\sigma}\left(\tau_{n}-\tau_{0}\right)$ where $\sigma$ is the (conserved) spin along the loop and the integer $n$ varies from 2 to $\infty$. Terms with $n=1$ correspond to anomalous diagrams and should be discarded. By summing over the different values of $n$, we obtain $\hat{\mathcal{G}}_{\sigma}^{(0)}\left(\mathbf{r}, \mathbf{r} ; \tau=0^{-}\right)-G_{\sigma}\left(\mathbf{r}, \mathbf{r} ; \tau=0^{-}\right)$where $\hat{\mathcal{G}}_{\sigma}^{(0)}$ is defined by Eq. (3.37). In the absence of particles in the UHB, both $\hat{\mathcal{G}}_{\downarrow}^{(0)}\left(\mathbf{r}, \mathbf{r} ; \tau=0^{-}\right)$and $G_{\downarrow}\left(\mathbf{r}, \mathbf{r} ; \tau=0^{-}\right)$are equal to zero. We conclude that closed loops of $\gamma_{\downarrow}$ particles vanish. On the other hand, since $\hat{\mathcal{G}}_{\uparrow}^{(0)}\left(\mathbf{r}, \mathbf{r} ; \tau=0^{-}\right)-G_{\uparrow}\left(\mathbf{r}, \mathbf{r} ; \tau=0^{-}\right)=n-1$, closed loops of $\gamma_{\uparrow}$ particles do not vanish, except when the density of particles $n$ equals 1 (half-filled case). 


\section{APPENDIX E: GREEN'S FUNCTION OF THE "PHYSICAL" FERMIONS}

In this section, we relate the "physical" Green's function $-\left\langle c_{a} c_{b}^{*}\right\rangle$ to the propagator of the $\gamma$ field. Consider the fermionic field $\phi$ defined in the spin reference frame determined by $\Omega$ [Eq. (B1)]:

$$
c_{\mathbf{r} \sigma}=\sum_{\sigma^{\prime}}\left(R_{\mathbf{r}}\right)_{\sigma \sigma^{\prime}} \phi_{\mathbf{r} \sigma^{\prime}}
$$

Since $\phi \equiv \gamma$ (in the space spanned by $\mathcal{B}_{\Omega_{\mathrm{r}}}^{\prime}$ ), we deduce

$$
-\left\langle c_{\mathbf{r} \sigma}(\tau) c_{\mathbf{r}^{\prime} \sigma}^{*}\left(\tau^{\prime}\right)\right\rangle=-\sum_{\sigma_{1}, \sigma_{2}}\left\langle\left(R_{\mathbf{r}}(\tau)\right)_{\sigma \sigma_{1}} \gamma_{\mathbf{r} \sigma_{1}}(\tau) \gamma_{\mathbf{r}^{\prime} \sigma_{2}}^{*}\left(\tau^{\prime}\right)\left(R_{\mathbf{r}^{\prime}}^{\dagger}\left(\tau^{\prime}\right)\right)_{\sigma_{2} \sigma}\right\rangle
$$

For fermions in the LHB, we then find

$$
\mathcal{G}_{\sigma}^{\mathrm{LHB}}\left(\mathbf{r} \tau, \mathbf{r}^{\prime} \tau^{\prime}\right)=-\left\langle\left(R_{\mathbf{r}}(\tau)\right)_{\sigma \uparrow} \gamma_{\mathbf{r} \uparrow}(\tau) \gamma_{\mathbf{r}^{\prime} \uparrow}^{*}\left(\tau^{\prime}\right)\left(R_{\mathbf{r}^{\prime}}^{\dagger}\left(\tau^{\prime}\right)\right)_{\uparrow \sigma}\right\rangle .
$$

Here $\gamma_{\uparrow}=e^{*} p f_{\uparrow}, \gamma_{\downarrow}=p^{*} d f_{\downarrow}$ and the mean values are to be taken with the action $S[f, e, p, d, \lambda ; \boldsymbol{\Omega}]$ [Eq. (2.34)]. The Green's function $\mathcal{G}^{\mathrm{LHB}}$ can be expressed as the functional derivative of the partition function calculated in the presence of external sources. It is then possible to carry out the steps leading to the effective action $S\left[\gamma^{*}, \gamma ; \boldsymbol{\Omega}\right]$ [Eqs. (3.21) and (3.24)]. Taking the functional derivative, one finds that $\mathcal{G}^{\mathrm{LHB}}$ is given by Eq. (E3) where the $\gamma$ field is now an elementary field and the mean value has to be taken with the action $S\left[\gamma^{*}, \gamma ; \boldsymbol{\Omega}\right]$.

${ }^{1}$ J. Hubbard, Proc. R. Soc. London, Ser. A 276, 238 (1963).

2 M.C. Gutzwiller, Phys. Rev. Lett. 10, 159 (1963).

${ }^{3}$ J. Kanamori, Prog. Theor. Phys. 30, 275 (1963).

${ }^{4}$ For a general introduction to the Hubbard model, see F. Gebhard, The Mott metal-insulator transition (SpringerVerlag, 1997).

${ }^{5}$ See, for instance, J. Voit, Rep. Prog. Phys. 57, 977 (1994).

${ }^{6}$ For a review, see A. Georges, G. Kotliar, W. Krauth, and M.J. Rozenberg, Rev. Mod. Phys. 68, 13 (1996).

${ }^{7}$ J.R. Schrieffer, X.G. Wen and S.C. Zhang, Phys. Rev. B 39, 11663 (1989).

${ }^{8}$ N.D. Mermin and H. Wagner, Phys. Rev. Lett. 17, 1133 (1966).

9 A. Kampf and J.R. Schrieffer, Phys. Rev. B 41, 6399 (1990); Phys. Rev. B 42, 7967 (1990);

${ }^{10}$ P. Montoux and D. Pines, Phys. Rev. B 47, 6069 (1993); B.L. Altshuler, L.B. Ioffe and A.J. Millis, Phys. Rev. B 52, 5563 (1995); J. Schmalian, D. Pines and B. Stojković, Phys. Rev. Lett. 80, 3839 (1998).

${ }^{11}$ N.E. Bickers, D.J. Scalapino and S.R. White, Phys. Rev. Lett. 62, 961 (1989); N.E. Bickers and D.J. Scalapino, Annals of Physics 193, 206 (1989).

12 N.E. Bickers and S.R. White, Phys. Rev. B 43, 8044 (1991).

13 N.E. Bickers, Int. J. Mod. Phys. B 5, 253 (1991).

${ }^{14}$ Y.M. Vilk and A.-M.S. Tremblay, J. Phys. I (France) 7, 1309 (1997).

${ }^{15}$ N.F. Mott, Metal-Insulator Transitions, 2nd edition (Taylor and Francis, London, 1990).

${ }^{16}$ J. Hubbard, Proc. R. Soc. London, Ser. A 296, 82 (1966).

${ }^{17}$ L.N. Bulaevskii and D.I. Khomskii, Soviet Physics-Solid State 14, 3015 (1973).
${ }^{18}$ D. Hone and P. Pincus, Phys. Rev. B 7, 4889 (1973); G. Beni, P. Pincus, and D. Hone, Phys. Rev. B 8, 3389 (1973).

${ }^{19}$ M. Plischke, J. Stat. Phys. 11, 159 (1974).

${ }^{20}$ K. Kubo, Prog. Theor. Phys. 64, 758 (1980); K. Kubo and M. Tada, Prog. Theor. Phys. 69, 1345 (1983); Prog. Theor. Phys. 71, 479 (1984).

${ }^{21}$ B.H. Zhao et al., Phys. Rev. B 36, 2321 (1987).

${ }^{22}$ K.K. Pan and Y.L. Wang, Phys. Rev. B 433706 (1991).

${ }^{23}$ M. Bartkowiak and K.A. Chao, Phys. Rev. B 46, 9228 (1992).

24 Yu.A. Izyumov, B.M. Letfulov, E.V. Shipitsyn, M. Bartkowiak, and K.A. Chao, Phys. Rev. B 46, 15697 (1992).

25 S. Pairault, D. Sénéchal, and A.-M.S. Tremblay, Phys. Rev. Lett. 80, 5389 (1998); Eur. Phys. J. B 16, 85 (2000).

${ }^{26}$ See also W. Metzner, Phys. Rev. B 43, 8549 (1991).

${ }^{27}$ P.W. Anderson, Phys. Rev. 115, 2 (1959).

${ }^{28}$ L.N. Bulaevskii, JETP 24, 154 (1967); M. Takahashi, J. Phys. C 10, 1289 (1977).

${ }^{29}$ A.B. Harris and R.V. Lange, Phys. Rev. 157 (1967); K.A. Chao, J. Spalek, and A.M. Oleś, Phys. Rev. B 18, 3453 (1978); J.E. Hirsch, Phys. Rev. Lett. 54, 1317 (1985); C. Gros, R. Joynt, and T.M. Rice, Phys. Rev. B 36, 381 (1987).

${ }^{30}$ D.P. Arovas and A. Auerbach, Phys. Rev. B 38, 316 (1988); S. Sarker, C. Jayaprakash, H.R. Krishnamurthy and M. Ma, Phys. Rev. B 40, 5028 (1989); D. Yoshioka, J. Phys. Soc. Japan 58, 3733 (1989).

${ }^{31}$ C. Jayaprakash, H.R. Krishnamurthy and S. Sarker, Phys. Rev. B 40, 2610 (1989); D. Yoshioka, J. Phys. Soc. Japan 58, 1516 (1989); A. Auerbach and D.E. Larson, Phys. Rev. B 43, 7800 (1991).

${ }^{32}$ In particular, charge carrier motion and spin fluctuations are essentially uncoupled at the mean-field level. Presumably, a correct description of their mutual interaction would require to go beyond mean-field theory. 
${ }^{33}$ See, for instance, M.U. Ubbens and P.A. Lee, Phys. Rev. B 49, 6853 (1994).

${ }^{34}$ A. Auerbach and B.E. Larson, Phys. Rev. Lett. 66, 2262 (1991).

${ }^{35}$ H.J. Schulz, Phys. Rev. Lett. 65, 2462 (1990); H.J. Schulz in The Hubbard Model, edited by D. Baeriswyl et al. (Plenum Press, New York, 1995).

${ }^{36}$ See also Z.Y. Weng, C.S. Ting, and T.K. Lee, Phys. Rev. B 43, 3790 (1991).

${ }^{37}$ Here we use our notations rather than those of Ref. 35.

${ }^{38}$ N.E. Bickers and D.J. Scalapino, cond-mat/0010480.

${ }^{39}$ N. Dupuis and S. Pairault, Int. J. Mod. Phys. B 14, 2529 (2000).

40 S.K. Sarker, J. Phys. C 21, L667 (1988).

${ }^{41}$ N. Dupuis, cond-mat/0105xxx.

${ }^{42}$ N. Dupuis, unpublished.

${ }^{43}$ A. Auerbach, Interacting Electrons and Quantum Magnetism (Springer Verlag, New York, 1994).

${ }^{44}$ G. Kotliar and A.E. Ruckenstein, Phys. Rev. Lett. 57, 1362 (1986).

45 J. Hubbard, Proc. Roy. Soc. (London) 285, 542 (1965).

${ }^{46}$ J.W. Negele and H. Orland, Quantum Many Particle Systems (Addison-Wesley, 1988).

47 The origin of the infinite normalization constant $\mathcal{N}^{M}$ $(M \rightarrow \infty)$ can be traced back by considering Eq. A3. At this stage, it is still possible to compute exactly the partition function. For the contribution of the singly occupied state, the integration over $\boldsymbol{\Omega}_{k}$ gives the factor $\int\left(\prod_{k=1}^{M} \frac{d \boldsymbol{\Omega}_{k}}{4 \pi}\right) \prod_{k=1}^{M}\left\langle\boldsymbol{\Omega}_{k} \mid \boldsymbol{\Omega}_{k-1}\right\rangle=2^{-M+1}$ so that we obtain $e^{\beta \mu} \frac{\mathcal{N}^{M}}{2^{M-1}\left(\alpha_{e} \alpha_{d}\right)^{M}}=2 e^{\beta \mu}$ which is the correct result. Thus the infinite normalization constant is suppressed when "spin fluctuations" are properly taken into account. In the context of spin-coherent-state path integrals, this issue has recently been considered by M. Stone, K.-S. Park, and A. Garg, cond-mat/0004247.

${ }^{48}$ M.V. Berry, Proc. R. Soc. Lond. A 392, 45 (1984); A. Shapere and F. Wilczek, Geometric phases in physics (World Scientific, 1989).

${ }^{49}$ For a spin-rotation-invariant slave-boson formulation based on the operator formalism, see T. Li, P. Wölfle, and P.J. Hirschfeld, Phys. Rev. B 40, 6817 (1989).

50 This result is obtained by noting that $\operatorname{det}\left(-G^{-1}\right)=$ $\int \mathcal{D}[\psi] \exp \left(\sum_{a, b} \psi_{a}^{*} G_{a b}^{-1} \psi_{b}\right)=Z_{\text {at }}^{(0)} e^{-S_{B}[\Omega]}$ to first order in $A^{0}$ (in a cumulant expansion).

${ }^{51}$ Equations of motion for Grassmann variables can be justified a posteriori by noting that they correctly reproduce the correlation functions: see J. Zinn-Justin, Quantum Field Theory and Critical Phenomena, Chap. 3.6 (Clarendon Press, Oxford, 1996).

${ }^{52}$ This identification requires-to perform a particle-hole transformation on the $\gamma_{\uparrow}$ field. 39

${ }^{53}$ P. Fulde, Electron Correlations in Molecules and Solids (Springer Verlag, Berlin, 1991).

${ }^{54}$ Eq. 3.35 is more commonly written as $c_{\mathbf{r} \sigma}^{\mathrm{LHB}}=b_{\mathbf{r} \sigma} h_{\mathbf{r}}^{*}$ where the bosonic field (Schwinger boson) $b_{\mathbf{r} \sigma}=z_{\mathbf{r} \sigma}(1-$ $\left.h_{\mathbf{r}}^{*} h_{\mathbf{r}} / 2\right)$ satisfies $\left|b_{\mathbf{r} \uparrow}^{2}\right|+\left|b_{\mathbf{r} \downarrow}^{2}\right|+h_{\mathbf{r}}^{*} h_{\mathbf{r}}=1$, with $h_{\mathbf{r}}^{*}=\gamma_{\mathbf{r} \uparrow}$ and $h_{\mathbf{r}}=\gamma_{\mathbf{r} \uparrow}^{*}$.

${ }^{55}$ For a half-filled system, there is no difference (as far as the free energy is concerned) between a $t / U$ expansion and an expansion around the atomic limit (i.e. a $t$ expansion).

${ }^{56}$ See also the discussion in Ref. 38

${ }^{57}$ P.A. Lee, Phys. Rev. Lett. 63, 680 (1989). 